\title{
Rational Hedging and Valuation of Integrated Risks under Constant Absolute Risk Aversion
}

\author{
DIRK BECHERER \\ Imperial College London \\ London SW7 2AZ, United Kingdom \\ dirk.becherer(@)imperial.ac.uk
}

first draft July 31, 2001; this version: May 1, 2003;

Please see

Insurance: Mathematics \& Economics 33 (1), 1-28, 2003, for the final published version. 


\title{
Rational Hedging and Valuation of Integrated Risks under Constant Absolute Risk Aversion
}

first draft July 31, 2001; this version: May 1, 2003;

\section{Dirk Becherer ${ }^{1}$}

\begin{abstract}
We study a rational valuation and hedging principle for contingent claims which integrate tradable and non-tradable sources of risk. The principle is based on the preferences of a rational investor with constant absolute risk aversion, and uses exponential utility indifference arguments. Properties of this valuation and of a corresponding hedging strategy are analyzed in a general semimartingale market framework. To obtain further constructive results and properties, a more specific class of semi-complete product models is studied in detail. This yields a computation scheme, simple valuation bounds, and results on diversification and information effects.
\end{abstract}

JEL: D81, G10, G22. $\quad$ MSC 2000: 60H30, 91B16, 91B28, 91B30.

Key words and phrases: Utility-indifference price, utility based hedging, exponential premium principle, diversification, integrated risks

\section{Introduction}

In recent years, there has been a growing interest, both on the academic and on the business side, in various financial products that incorporate different kinds of risks. Examples include catastrophe bonds, equity linked life insurance, credit and default risky securities, alternative risk transfers, or weather derivatives. For an overview and further references, see Klüppelberg (2001).

From a methodologist's point of view, those products are interesting because they integrate risks from qualitatively different sources, e.g. classical actuarial risk and financial market risk. On the one hand, the respective risky future payoff is related to the evolution of assets that are liquidly traded in financial markets. This relation can be either directly specified by the contract but may also be more indirect if the payoff is just stochastically correlated to tradable assets. For instance, it seems plausible that catastrophic events or credit defaults are statistically related to liquidly

\footnotetext{
${ }^{1}$ Department of Mathematics, Imperial College, London SW7 2AZ, UK; Email: dirk.becherer(@)imperial.ac.uk Financial support by the German Science Foundation via the National Research Center SFB 373 "Quantification and Simulation of Economic Processes", Humboldt University Berlin, and via Graduiertenkolleg "Stochastic Processes and Probabilistic Analysis", Technical University Berlin, is gratefully acknowledged. This article is based on a part of my thesis. I thank Martin Schweizer and the referee for helpful comments.
} 
traded indices of certain industries or to stock prices of individual firms. This should allow an issuer to hedge at least a part of the risk by trading in those assets. On the other hand, the payoff of such products typically is not purely dependent on liquidly tradable securities so that, for principal reasons, such risks cannot be hedged perfectly but only partially. Some residual risk always remains with the issuer. The natural modelling framework for such problems is provided by incomplete market models which incorporate both tradable and non-tradable factors of risk, whereas complete models like the Black-Scholes model are inappropriate.

The occurrence of products which integrate qualitatively different risks underlines the need for integrated theoretical methods to evaluate and hedge them in a consistent manner. Advocated by Bühlmann (1987), this has lead to a convergence of the formerly distinct areas of insurance and finance; see Bühlmann (1983), Delbaen and Hazendonck (1989), Gerber and Shiu (1996), Geman (1999), Embrechts (2000), or Schweizer (2001) among others. For consistency reasons, it is reasonable to rely on one underlying principle for the valuation and hedging of integrated risks. In this paper, we use the utility-indifference approach with respect to exponential utility, which corresponds to the preferences of a rational investor with constant absolute risk aversion. The approach can be seen as an adaption of the (static) actuarial exponential premium principle to a dynamical financial market setting, and appeared first in Hodges and Neuberger (1989). At present, the structure of the mathematical problem is well characterized on an abstract level, cf. Cvitanić et al. (2001), Frittelli (2000a), Delbaen et al. (2002), Owen (2002), or Rouge and El Karoui (2000). But more explicit results on the utility-indifference valuation and on the hedging strategy have been available so far basically in a Brownian model world with a geometric Brownian motion describing the tradable asset; see Davis (2000), Henderson and Hobson (2002), Henderson (2002), Musiela and Zariphopoulou (2002a), and the overview in Delbaen et al. (2002). For recent results in models which involve point processes, see Becherer (2002) and Young and Zariphopoulou (2002). Related approaches relying on quadratic indifference criteria have been studied by Møller (2001, 2003a,b) and Schweizer (2001).

The aim of the present paper is twofold. First, we present, on a slightly more general level and in a form which explicitly accounts for the additional liability, recent martingale duality results for the indifference valuation and hedging problem with respect to exponential utility. We survey and show, respectively, that several nice properties still hold in a general dynamic market framework. Secondly, we study a more specific class of semi-complete product models to obtain more constructive results and further original properties, concerning diversification and information effects. The paper is organized as follows. Section 2.1 describes the general framework where risky securities prices evolve as semimartingales. We formulate the utility-indifference hedging and pricing problem in Section 2.3, building on the martingale duality foundations from Section 2.2. The utilityindifference price $\pi$ of a contingent claim is defined from the perspective of an issuer with constant absolute risk aversion, such that an issuer who takes the risky future liabilities from the claim is just compensated in terms of maximal expected utility if he receives the premium $\pi$. In passing through the paper, we will show that such a valuation basically inherits all "desirable properties" (cf. Gerber (1979)) from the static exponential premium principle. Besides, it is also consistent 
with the no-arbitrage principle of mathematical finance, cf. Frittelli (2000a), and can be seen a convex measure of risk in the sense of Föllmer and Schied (2002). Moreover, the utility-indifference price is a systematic interpolation between the totally risk-averse super-replication value and a rather risk-neutral valuation which relies on a zero marginal rate of substitution argument. We define the utility-indifference hedging strategy as the optimal adjustment of the portfolio strategy for an investor who has issued the claim. Building on results from Grandits and Rheinländer (2002), the solutions to the utility-indifference pricing and hedging problem and also to the utility maximization problem under additional liability are characterized in Section 3 by a stochastic representation problem. Solving the problem amounts to finding an equivalent martingale measure whose density has a specific form. This provides a duality verification method for further results, and the density process of the measure induces the definition of an indifference valuation process. The latter has an economic interpretation, related to the certainty equivalent of the effective liability that remains with the issuer after an optimal partial hedge. In Section 4, we turn to a more specific class of models which are composed by a complete financial sub-market and by a countable number of additional independent factors of randomness, representing non tradable sources of risk. This framework in Section 4.1 is flexible enough to study several interesting problems, but it is still quite explicitly tractable since the set of equivalent martingale measures for the dual problem has a convenient structure, explored in Section 4.2. Section 4.3 provides simple upper and lower bounds for the utility-indifference price. If new information about non-market sources of risk only comes in at discrete times, the utility-indifference price can be calculated by an explicit backward computation scheme. Moreover, we show that the utility-indifference valuation decreases when the issuer's information about the non-tradable factors of risk improves by, say, better monitoring or reporting. Section 4.4 proves that the utility-indifference price (resp. hedging strategy) for an aggregated amount of contingent claims is given by the sum of the corresponding prices (resp. hedging strategies) for the individual claims, whenever those claims are conditionally independent given the information of the complete sub-market. Under similar assumptions, we finally show how diversification effects among a large number of individual risks can asymptotically lead to a utility based valuation for the aggregate which is risk neutral with respect to non-tradable factors of risk under the objective probability measure $P$.

\section{Preliminaries}

In this section, we set up the general modelling framework for this paper. Before we formulate the utility-indifference valuation and hedging problem in Section 2.3, we summarize some key martingale duality results on exponential utility maximization, following Delbaen et al. (2002) and Kabanov and Stricker (2002). We take this as an opportunity to include some slight but useful generalizations, and to give a presentation which is tailored for our later purposes. 


\subsection{General semimartingale framework}

Our mathematical framework is given by a probability space $(\Omega, \mathcal{F}, P)$, a finite time horizon $T$ and a filtration $\mathbb{F}=\left(\mathcal{F}_{t}\right)_{0 \leq t \leq T}$ satisfying the usual conditions of right-continuity and completeness. For simplicity let $\mathcal{F}_{0}$ be trivial and $\mathcal{F}_{T}=\mathcal{F}$. On occasion, we will introduce further filtrations. All semimartingales with respect to a filtration that satisfies the usual conditions are taken to have right continuous paths with left limits. Expectations are taken with respect to $P$ unless specified otherwise. Let $S=\left(S^{i}\right)_{i=1, \ldots, d}$ be an $\mathbb{R}^{d}$-valued $(P, \mathbb{F})$-semimartingale. We consider $S$ as the discounted prices of the tradable risky assets in a financial market which contains a risk-less asset with discounted price constant at 1 . Throughout, we make the

Standing Assumption: $S$ is $\mathbb{F}$-locally bounded.

The sets of absolutely continuous and equivalent (local) martingale measures for $S$ with respect to $\mathbb{F}$, and those with finite relative entropy are defined as

$$
\begin{aligned}
& \mathbb{P}_{a}:=\{Q \ll P \mid S \text { is a local }(Q, \mathbb{F}) \text {-martingale }\} \\
& \mathbb{P}_{e}:=\{Q \sim P \mid S \text { is a local }(Q, \mathbb{F}) \text {-martingale }\} \\
& \mathbb{P}_{f}:=\mathbb{P}_{f}(P):=\left\{Q \in \mathbb{P}_{a} \mid H(Q \mid P)<\infty\right\}
\end{aligned}
$$

where $H(Q \mid P)=E\left[\frac{d Q}{d P} \log \frac{d Q}{d P}\right]$ denotes the relative entropy of $Q$ with respect to $P$, which is always non negative (cf. Ihara (1993)). Intuitively speaking, the relative entropy measures how close a probability distribution is to another one. We assume throughout that our financial model is free of arbitrage (cf. Delbaen and Schachermayer (1994)) in the sense that

$$
\mathbb{P}_{e} \cap \mathbb{P}_{f} \neq \emptyset,
$$

and denote by $\Theta$ some set of "permitted" trading strategies. Technically, $\Theta$ is a cone in the space $L(S)$ of predictable and $S$-integrable processes. We refrain from a concrete specification of $\Theta$ because Delbaen et al. (2002) and Schachermayer (2002) emphasize that there are many possible choices for $\Theta$ which lead to the same maximal expected utility function $u$. Technically, this is related to the fact that for several choices of $\Theta$ the martingale approach to the (primal) utility maximization problem leads to the same dual problem, and to the same value function $u$. Since both the utility-indifference price and the corresponding hedging strategy will be defined via $u$, it is reasonable to rely on the more robust dual side of the optimization problem (see below) but not on a specific choice of $\Theta$ to obtain more universal results. We will also see that the duality gives us a powerful tool to prove many properties of the utility-based pricing and hedging method, because the dual side provides a convenient path to the primal problem; see Rogers (2001) for a recent survey on martingale duality approaches.

\subsection{Duality results on exponential utility optimization}

We need some preparation in order to formulate this robust duality. To this end, let $B$ denote some random variable and fix some $\alpha \in(0, \infty)$. If $\exp (\alpha B)$ is $P$-integrable we can define a measure 
$d P_{B}:=\left(E\left[e^{\alpha B}\right]\right)^{-1} e^{\alpha B} d P$. In the sequel, it is sometimes convenient to work under $P_{B}$ instead of $P$. Since $P_{B} \sim P$ we could replace $P$ by $P_{B}$ in the definitions of $\mathbb{P}_{a}$ and $\mathbb{P}_{e}$ without altering these sets of measures (although the densities with respect to $P_{B}$ are different from those with respect to $P$ ). Concerning $\mathbb{P}_{f}$, the situation is less trivial, and we need some further assumptions to obtain

$$
\mathbb{P}_{f}(P)=\mathbb{P}_{f}\left(P_{B}\right)
$$

Delbaen et al. (2002) considered the case where $B$ is bounded from below. For random variables $B$ that are unbounded from below, Lemma A.1 yields that

$$
E\left[e^{(\alpha+\varepsilon) B}\right]<\infty \text { and } E\left[e^{-\varepsilon B}\right]<\infty \text { for some } \varepsilon>0
$$

implies that $B$ is in $L^{1}(Q)$ for all $Q \in \mathbb{P}_{f}(P)$, and that (2.3) holds. We can then simply write $\mathbb{P}_{f}$ without any ambiguity with respect to $P$ or $P_{B}$. We will now specify conditions on $\Theta$ that are used in the sequel. We mostly suppose that for $\Theta$ the following robust duality holds:

$$
\begin{gathered}
\sup _{\Theta} E\left[-\exp \left(-\alpha\left(\int_{0}^{T} \vartheta d S-B\right)\right)\right]=-\exp \left(\sup _{Q \in \mathbb{P}_{a}}\left\{E_{Q}[\alpha B]-H(Q \mid P)\right\}\right) \\
\text { holds for any } \alpha>0 \text { and } B \text { satisfying }(2.4) .
\end{gathered}
$$

Occasionally, we will also require that

$$
\Theta \text { contains } \Theta_{\mathcal{M}}:=\left\{\vartheta \in L(S) \mid \vartheta \cdot S \text { is a }(Q, \mathbb{F}) \text {-martingale for all } Q \in \mathbb{P}_{f}\right\} .
$$

For appropriate spaces $\Theta$, both properties constitute key duality results from Delbaen et al. (2002) and Kabanov and Stricker (2002): Equation (2.5) states that the value of the utility optimization problem over $\Theta$ is given by value of the corresponding dual problem over the set $\mathbb{P}_{a}$, and (2.6) ensures that the optimal investment strategy is attained in $\Theta$.

Example 2.1 Consider the following spaces of strategies: $\Theta_{1}:=\left\{\vartheta \in L(S) \mid \vartheta \cdot S\right.$ is a $Q^{B_{-}}$ martingale $\}$ (with $Q^{B}$ given by Prop. 2.2), $\Theta_{2}:=\Theta_{\mathcal{M}}, \Theta_{3}:=\{\vartheta \in L(S) \mid \vartheta \cdot S$ is bounded $\}$, or $\Theta_{4}:=\left\{\vartheta \in L(S) \mid(\vartheta \cdot S)^{-}\right.$is bounded $\}$. Then (2.5) is satisfied for $\Theta_{1}, \Theta_{2}, \Theta_{3}$, and $\Theta_{4}$ (see Delbaen et al. (2002), Schachermayer $(2002,2001))$; while (2.6) is satisfied for $\Theta_{2}$ by definition and thus also for $\Theta_{1} \supseteq \Theta_{2}$.

In the next proposition we recall central duality results from Delbaen et al. (2002) and Kabanov and Stricker (2002) on the exponential utility maximization problem

$$
u(x-B):=u(x-B ; \alpha):=\sup _{\vartheta \in \Theta} E\left[-\exp \left(-\alpha\left(x+\int_{0}^{T} \vartheta d S-B\right)\right)\right]
$$

and the corresponding dual problem

$$
\sup _{Q \in \mathbb{P}_{f}}\left\{\alpha E_{Q}[B]-H(Q \mid P)\right\} .
$$

Related results have also been obtained by several other authors. 
Proposition 2.2 Assume (2.2), (2.4) and (2.5). Then

1. There exists a unique $Q^{B} \in \mathbb{P}_{f} \cap \mathbb{P}_{e}$ which maximizes (2.8).

2. The density of $Q^{B}$ takes the form $\frac{d Q^{B}}{d P}=\exp \left(-\alpha\left(c^{B}+\int_{0}^{T} \vartheta^{B} d S-B\right)\right)$ for some $\vartheta^{B} \in L(S)$ such that $\vartheta^{B} \cdot S$ is a $Q^{B}$-martingale and $c^{B} \in \mathbb{R}$. Moreover, $\vartheta^{B}$ is in $\Theta_{\mathcal{M}}$.

3. The maximal expected utility in (2.7) is given by

$$
u(x-B ; \alpha)=-e^{-\alpha x} \exp \left(\sup _{Q \in \mathbb{P}_{f}}\left\{\alpha E_{Q}[B]-H(Q \mid P)\right\}\right),
$$

4. and $u(x-B ; \alpha)=E\left[-\exp \left(-\alpha\left(x+\int_{0}^{T} \vartheta^{B} d S-B\right)\right)\right]=-\exp \left(-\alpha\left(x-c^{B}\right)\right)$.

The measure $Q^{0}$ (corresponding to $B=0$ ) minimizes $H(Q \mid P)$ over $Q \in \mathbb{P}_{a}$ and is therefore called minimal entropy martingale measure. Although our assumptions on $B$ and on the space of strategies are slightly more general than in Delbaen et al. (2002) and Kabanov and Stricker (2002), it is straightforward to adapt their proofs. The details are relegated to the appendix.

\subsection{Utility-indifference pricing and hedging - formulation of the problem}

We now introduce our main objects of interest: a utility-based valuation and a corresponding hedging strategy for a risky future payoff.

Definition 2.3 If there is a unique solution $\pi(B)=\pi(B ; \alpha)$ to the equation

$$
u(x ; \alpha)=u(x+\pi-B ; \alpha),
$$

we call this solution the utility-indifference (selling) price for $B$.

In the absence of dynamical trading opportunities (this corresponds to letting $S \equiv S_{0}$ be constant) the utility-indifference price $\pi$ is defined as the solution of the equation

$$
U(x)=E[U(x+\pi-B)]
$$

with a utility function $U$ which is exponential in our setting, i.e. $U(x)=-\exp (-\alpha x)$. The solution $\pi$ to equation (2.10) yields a valuation method which has been known for a long time. In fact, the origins of the idea can be traced back to the 18th century when Daniel Bernoulli (1738) suggested that an investor, say gambler, will rank risky ventures, say lotteries, by their expected utilities. In actuarial mathematics, such a valuation method is known as the "premium principle of equivalent utility" (cf. Gerber and Parfumi (1998) for an overview) and has certain desirable properties if and only if the utility function $U$ is exponential (cf. Gerber (1979), chapter 5)

In the presence of a dynamical financial market, investors can maximize their expected utility and reduce risk from a terminal liability $B$ by dynamical trading. Taking this into account, leads to Definition 2.3 for the utility-indifference price. This adaptation of the classical idea from the static situation to the dynamic financial market case appeared first in Hodges and Neuberger (1989), and $\pi(B)$ can be interpreted as the adjustment to the investor's initial capital that compensates 
for the additional terminal liability $B$ in terms of maximal expected (exponential) utility. In this sense, $\pi(B)$ is a subjective fair valuation (premium) for the liability $B$ from the perspective of a risk averse investor and can be considered as a measure of risk, cf. Remark 3.1. One might also consider $\pi(B)$ in terms of acceptable financial positions as the minimal premium needed to achieve an acceptable financial position at terminal time. In fact,

$$
\pi(B)=\inf \left\{y \in \mathbb{R} \mid x+y+\int_{0}^{T} \theta d S-B \in \mathcal{A} \text { for some } \theta \in \Theta\right\}
$$

with $\mathcal{A}:=\{X \mid E[U(X)] \geq \ell\}$ being the set of acceptable positions for utility $U(x)=-\exp (-\alpha x)$ and acceptance level $\ell=u(x ; \alpha)$. But let us emphasize that in general $\pi(B)$ must not be considered as a price for which $B$ can be bought and sold in the financial market.

Remark 2.4 (Extensions) In the same spirit, one could define a corresponding utility-indifference buying price as the unique solution $\pi^{b}(B)$ to the equation $u(x)=u\left(x-\pi^{b}+B\right)$. It is easy to see that $\pi^{b}(B)=-\pi(-B)$ holds if the latter is defined. Furthermore, it may be relevant to consider the utility-indifference (selling) price of an additional claim $B$ for an issuer who already has a liability, say, $C$. One can verify that the unique solution $\pi(B \mid C)$ to the equation $u(x-C)=$ $u(x+\pi(B \mid C)-(B+C))$ is given by $\pi(B \mid C)=\pi(B+C)-\pi(C)$, provided the latter terms are defined. To see this, note that $u(x-C)=u(x-\pi(C))=u(x-\pi(C)+\pi(B+C)-(B+C))$ holds thanks to (3.2).

We now define the hedging strategy $\psi(B ; \alpha)$ as the adjustment of the optimal strategy without liability that is necessary to obtain a strategy that is optimal under the terminal liability $B$. More vaguely, it is the part of the optimal strategy $\vartheta^{B}$ that stems from the additional liability $B$.

Definition 2.5 Under the assumptions for Proposition 2.2, we define the utility-indifference hedging strategy $\psi$ for $B$ by

$$
\psi(B)=\psi(B ; \alpha):=\vartheta^{B, \alpha}-\vartheta^{0, \alpha} .
$$

Part 2 of Proposition 2.2 implies that $\vartheta^{B}$ and $\vartheta^{0}$ are unique in the sense that the processes $\int \vartheta^{B} d S$ and $\int \vartheta^{0} d S$, respectively, are unique. Hence, the hedging strategy is unique in the sense that $\int \psi S$ is unique. More formally, $\psi$ is defined in the quotient space of $L(S)$ with respect to the equivalence relation $\vartheta \sim \xi: \Leftrightarrow \int \vartheta d S=\int \xi d S$. The definition of $\psi$ can be extended similarly as the one for $\pi$, in analogy to Remark 2.4 .

Remark 2.6 (Relaxing the exponential moment condition)

In some applications, the integrability condition (2.4) will not be satisfied by the claim. To explain a straightforward way to relax this condition, let $\tilde{B}$ be such a claim - e.g. a call option $\tilde{B}=\left(S_{T}-K\right)^{+}$ in a Black-Scholes type model. Suppose there exists $\xi \in \Theta$ such that $B:=\tilde{B}-\int_{0}^{T} \xi d S$ satisfies (2.4) and that $\Theta$ is a linear space. Given that the assumptions for Proposition 2.2 hold, we can then apply our analysis for $B$ to obtain $\pi(\tilde{B} ; \alpha)=\pi(B ; \alpha)$, using the linearity of $\Theta$. Analogously, 
$\psi(\tilde{B})=\psi(B)+\xi$ can be taken as the utility-indifference hedging strategy for $\tilde{B}$. In the example, choosing $\xi=1$ for instance transforms $\widetilde{B}$ into a bounded claim $B=\left(S_{T}^{1}-K\right)^{+}-\left(S_{T}^{1}-S_{0}^{1}\right)$.

\section{General properties and verification methods}

Under the assumptions needed for Proposition 2.2, one obtains (see eq. (4.6) in Delbaen et al. (2002)) the following formula for the utility-indifference price

$$
\pi(B ; \alpha)=\sup _{Q \in \mathbb{P}_{f}}\left\{E_{Q}[B]-\frac{1}{\alpha}\left(H(Q \mid P)-H\left(Q^{0} \mid P\right)\right)\right\} .
$$

This yields almost directly that the following properties (3.2)-(3.7), shown by Frittelli (2000a) and Rouge and El Karoui (2000) under additional assumptions and in part by different methods, still hold in the present framework:

$$
\begin{aligned}
& \text { (Independence on initial capital) } \quad \pi(B ; \alpha) \text { does not depend on } x \text {, } \\
& \text { (Monotonicity in } \alpha) \quad \alpha \mapsto \pi(B ; \alpha) \text { is increasing in } \alpha \text {, } \\
& \text { (Volume-scaling) } \quad \pi(\beta B ; \alpha)=\beta \pi(B ; \beta \alpha) \text { for } \beta \in(0,1] \text {, and } \\
& \text { (Translation invariance) } \quad \pi(B+c ; \alpha)=\pi(B ; \alpha)+c \text { for } c \in \mathbb{R} \text {. }
\end{aligned}
$$

If fact, equation (3.4) holds for any $\beta \in(0, \infty)$ provided that both sides satisfy formula (3.1). For bounded random variables $B_{1}, B_{2}$ and $\lambda \in[0,1]$, formula (3.1) moreover yields

$$
\begin{array}{lc}
\text { (Convexity) } & \pi\left(\lambda B_{1}+(1-\lambda) B_{2} ; \alpha\right) \leq \lambda \pi\left(B_{1} ; \alpha\right)+(1-\lambda) \pi\left(B_{2} ; \alpha\right), \text { and } \\
\text { (Monotonicity) } & \pi\left(B_{1} ; \alpha\right) \leq \pi\left(B_{2} ; \alpha\right) \text { if } B_{1} \leq B_{2} .
\end{array}
$$

Remark 3.1 (Interpretation as a measure of risk)

By (3.5)-(3.7), the mapping $\varrho: X \mapsto \pi(-X ; \alpha)$ satisfies all axioms which constitute a convex measure of risk on the set of bounded random variables. For the notion and a discussion of such risk measures we refer to Föllmer and Schied (2002). Let us just note here that $\varrho$ assigns a monetary measure of risk to a financial position $X$, corresponding to a liability $B=-X$. This point of view is instructive for the understanding and interpretation of some subsequent results, like Corollary 3.3. In general, $\varrho$ is not additive or sub-additive; and it is a semantic question whether a risk measure should be so. A risk measure that is imposed by an external regulator, e.g. a supervising agency, should be sub-additive because otherwise a supervised entity could just split up to meet the requirements, cf. Artzner et al. (1999). However, for its own sake a firm might be well advised to view its aggregated risks in a risk-averse way which discriminates against correlated exposures and honors diversification - as the latter is fairly common sense. To penalize concentrated exposures with respect to specific risks, the properties of positive homogeneity and sub-additivity have to be dropped. As a consequence, the risk analysis of the aggregate becomes more complex since it cannot be done by parts in general. In principle, each individual risk has 
to be judged with respect to its contribution to the overall exposure, see Remark 2.4. However, individual risks which are sufficiently independent can be treated separately, see Section 4.4.

For any $B \in L^{\infty}(P)$ that is attainable in the sense that $B=b+\int_{0}^{T} \vartheta d S$ for $\vartheta \in L(S)$ with $\int \vartheta d S$ bounded (uniformly in $t$ ), we have $E_{Q}[B]=b$ for all $Q \in \mathbb{P}_{a}$. By (3.1) this yields elementary No-Arbitrage (NA) consistency of $\pi$ in the sense that

$$
\text { (Elementary NA-consistency) } \quad \pi\left(b+\int_{0}^{T} \vartheta d S ; \alpha\right)=b
$$

for $b \in \mathbb{R}$ and $\vartheta \in L(S)$ with $\int \vartheta d S$ bounded - implying $\pi(B ; \alpha)=E_{Q}[B]$ for all $Q \in \mathbb{P}_{a}$ (for such $B)$. Since $Q^{0}$ is in $\mathbb{P}_{e}$, Proposition 3.2 below moreover implies that the utility-indifference price of any bounded claim lies within the interval of possible arbitrage free valuations, that is

$$
\text { (NA-consistency) } \quad \inf _{Q \in \mathbb{P}_{e}} E_{Q}[B] \leq \pi(B ; \alpha) \leq \sup _{Q \in \mathbb{P}_{e}} E_{Q}[B] \quad \text { for } B \in L^{\infty}(P) \text {. }
$$

In this sense, consistency of $\pi$ with the No-Arbitrage principle also holds for non attainable claims. The latter was originally pointed out by Frittelli (2000a) in a related but slightly different context. As an aside, Proposition 3.2 also yields

$$
\text { (Nonnegative market-adjusted safety loading) } \quad \pi(B ; \alpha) \geq E_{Q^{0}}[B] .
$$

Comparing $\pi(B ; \alpha)$ with $E_{P}[B]$ would not make much sense in a dynamical financial market. To see this, observe that (bounded) attainable claims can be perfectly hedged by their replication costs which is the expectation of the payoff under a martingale measure $Q \in \mathbb{P}_{a}$ (cf. the remark before (3.8)), and is typically different from the expectation under the objective probability $P$. A notable exception where is makes sense to compare $\pi(B ; \alpha)$ with $E_{P}[B]$ is a situation where the claim $B$ incorporates only independent non-tradable sources of risk which can be separated from the financial market. For details, see (4.22) (there, $E_{P}[B]=E_{Q^{0}}[B]$ holds for any $\mathcal{I}_{T^{-m e a s u r a b l e}}$ claim $B$ ).

The integrability conditions subsequently imposed on $B$ in (3.12) hold if and only if $B \in L_{\exp }(P) \cap$ $L_{\exp }\left(Q^{0}\right)$, where $L_{\exp }(P):=\left\{X \mid E_{P}[\exp (\varepsilon|X|)]<\infty\right.$ for some $\left.\varepsilon>0\right\}$ denotes the Orlicz space. Both the conditions in (3.11) and in (3.12) are satisfied for $B \in L^{\infty}(P)$ in particular.

\section{Proposition 3.2 (risk-aversion asymptotics)}

Suppose (2.2) and (2.5) hold. Then $\alpha \mapsto \pi(B ; \alpha), \alpha \in(0, \infty)$, is non-decreasing and

$$
\begin{aligned}
\lim _{\alpha \uparrow \infty} \pi(B ; \alpha) & =\sup _{Q \in \mathbb{P}_{e}} E_{Q}[B] \quad \text { if (2.4) holds for all } \alpha, \\
\lim _{\alpha \downarrow 0} \pi(B ; \alpha) & =E_{Q^{0}}[B] \quad \text { if (2.4) holds for } \alpha \text { small enough, and } B \in L_{\exp }\left(Q^{0}\right) .
\end{aligned}
$$

Hence, the utility-indifference price tends to the super-replication price, which is equal to the supremum of the expectation of $B$ over all equivalent martingale measures, when the risk-aversion 
tends to infinity. For vanishing risk aversion, the utility-indifference price tends to a risk-neutral valuation under the minimal entropy martingale measure $Q^{0}$. Recalling Remark 2.4, one can see that the utility-indifference buying price $-\pi(-B ; \alpha)$ tends to the lower bound of the interval of arbitrage-free prices, that is the infimum of the expectation of $B$ over all martingale measures, when the risk aversion tends to infinity. Rouge and El Karoui (2000) proved these asymptotics for bounded claims in a Brownian setting, using methods from the theory of backward stochastic differential equations. For the locally bounded semimartingale framework, the limit (3.11) was proven by Delbaen et al. (2002) (cf. the proof of their Prop. 11) and it remains to show (3.12) here. Independently of this work, Stricker (2002) has recently found an alternative proof for (3.12) under a slightly weaker integrability condition, using the Fenchel inequality.

Proof of Proposition 3.2: From (3.1) and (3.3) follows that $\pi(B ; \alpha) \geq E_{Q^{0}}[B]$ for all $\alpha$ and that $\pi(B ; \alpha)$ decreases for decreasing $\alpha$. Hence,

$$
E_{Q^{0}}[B] \leq \lim _{\alpha \downarrow 0} \pi(B ; \alpha)=\inf _{\alpha>0} \sup _{Q \in \mathbb{P}_{f}}\left\{E_{Q}[B]-\frac{1}{\alpha}\left(H(Q \mid P)-H\left(Q^{0} \mid P\right)\right)\right\} .
$$

Suppose $E_{Q^{0}}[B]+\delta \leq \lim _{\alpha \downarrow 0} \pi(B ; \alpha)$ for some $\delta>0$. Then we could find for any $\alpha$ small enough some $Q_{\alpha} \in \mathbb{P}_{f}$ such that

$$
\left(E_{Q_{\alpha}}[B]-E_{Q^{0}}[B]\right)-\frac{1}{\alpha}\left(H\left(Q_{\alpha} \mid P\right)-H\left(Q^{0} \mid P\right)\right)>\frac{\delta}{2} .
$$

Using the inequality $E_{Q_{\alpha}}[|B|] \leq \frac{1}{\varepsilon}\left(H\left(Q_{\alpha} \mid P\right)+\frac{1}{e} E_{P}\left[e^{\varepsilon|B|}\right]\right)$ (cf. (A.2)), we then obtain that

$$
\text { const }+\left(\frac{1}{\varepsilon}-\frac{1}{\alpha}\right)\left(H\left(Q_{\alpha} \mid P\right)-H\left(Q^{0} \mid P\right)\right)>\frac{\delta}{2}
$$

where const $=\frac{1}{\varepsilon}\left(\frac{1}{e} E_{P}\left[e^{\varepsilon|B|}\right]+H\left(Q^{0} \mid P\right)\right)-E_{Q^{0}}[B]$ is nonnegative, finite (for some $\varepsilon>0$ ), and does not depend on $\alpha$. As the term in the second bracket of (3.14) is nonnegative for all $\alpha$, the inequality implies

$$
\lim _{\alpha \downarrow 0}\left(H\left(Q_{\alpha} \mid P\right)-H\left(Q^{0} \mid P\right)\right)=0 .
$$

and therefore $H\left(Q_{\alpha} \mid Q^{0}\right) \rightarrow 0$ since $H\left(Q_{\alpha} \mid P\right) \geq H\left(Q_{\alpha} \mid Q^{0}\right)+H\left(Q^{0} \mid P\right)$ by Theorem 2.2 of Csiszár (1975). In particular, $Q_{\alpha}$ converges to $Q^{0}$ in entropy, therefore in total variation, and

$$
E_{Q_{\alpha}}[B] \rightarrow E_{Q^{0}}[B] \quad \text { for } \alpha \downarrow 0 .
$$

The latter is evident for $B \in L^{\infty}$. We next show it for $B \in L_{\exp }\left(Q^{0}\right)$. By a classical variational inequality for the relative entropy (Theorem 1.4.4 in Ihara (1993), plus monotone convergence),

$$
E_{Q_{\alpha}}\left[|B| 1_{\{|B|>c\}}\right] \leq \frac{1}{\varepsilon}\left(H\left(Q_{\alpha} \mid Q^{0}\right)+\log E_{Q^{0}}\left[\exp \left(\varepsilon|B| 1_{\{|B|>c\}}\right)\right]\right)
$$

holds for all random variables $B$ and $c \geq 0, \varepsilon>0$. By hypothesis, there is $\varepsilon>0$ such that $\exp (\varepsilon|B|) \in L^{1}\left(Q^{0}\right)$. For $\alpha \downarrow 0$ and $c \uparrow \infty$ the right hand side of (3.17) tends to zero. It follows that (3.16) also holds for non-bounded random variables $B \in L_{\exp }\left(Q^{0}\right)$. We conclude that the 
limes inferior for $\alpha \downarrow 0$ of the left hand side in (3.13) is less than or equal to 0. Clearly, this is a contradiction which proves the claim.

In view of the volume scaling property (cf. (3.4) plus subsequent remark), the limits in Proposition 3.2 can be rewritten as volume-asymptotics of the utility-indifference price. This yields

Corollary 3.3 (volume asymptotics) Suppose (2.2) and (2.5) hold. For any $\alpha \in(0, \infty)$, the mapping $\beta \mapsto \frac{1}{\beta} \pi(\beta B ; \alpha), \beta \in(0, \infty)$, is non-decreasing and

$$
\begin{aligned}
& \lim _{\beta \uparrow \infty} \frac{1}{\beta} \pi(\beta B ; \alpha)=\sup _{Q \in \mathbb{P}_{e}} E_{Q}[B] \quad \text { if (2.4) holds for all } \alpha, \\
& \lim _{\beta \downarrow 0} \frac{1}{\beta} \pi(\beta B ; \alpha)=E_{Q^{0}}[B] \quad \text { if (2.4) holds for } \alpha \text { small enough, and } B \in L_{\exp }\left(Q^{0}\right) .
\end{aligned}
$$

The appearing quantity $\frac{1}{\beta} \pi(\beta B ; \alpha)$ can be interpreted as price per unit for a given amount (volume) $\beta$ of claims $B$. It is increasing in $\beta$ since $\pi$ is defined as a risk-averse and subjective fair valuation from the issuer's perspective. For concreteness, consider an insurance company that insures skyscrapers against earthquakes and has already many clients in San Francisco. Adding a further client there, would increase the volume of the insured risk that is exposed to the next big earthquake in northern California. Provided the insurer has to keep its risks and cannot resell them, he would prefer - ceteris paribus - an in other respects comparable risk elsewhere. That is, his risk-averse valuation of an additional earthquake-related insurance risk is higher when it increases his already large exposure to the next big earthquake in California.

Remark 3.4 (Relation to the 'zero marginal rate of substitution'-price)

Suppose $B$ is bounded, $\alpha$ equals 1 , and $\Theta_{\mathcal{M}}$ is a subset of $\Theta$. By Parts 2 and 4 of Proposition 2.2, we have

$$
E_{Q^{0}}[B]=E_{P}\left[e^{-c^{0}-\int_{0}^{T} \vartheta^{0} d S} B\right]=\frac{E_{P}\left[U^{\prime}\left(x+\int_{0}^{T} \vartheta^{0} d S\right) B\right]}{\frac{\partial}{\partial x}\left(-e^{-\left(x-c^{0}\right)}\right)}
$$

with $U(x):=-e^{-x}$. By formula (3) in Davis (1997), this shows that $E_{Q^{0}}[B]$ equals Davis' so-called "fair price" $\widehat{p}$ for $B$ with respect to the exponential utility function $U$. The definition of $\widehat{p}$ relies on a classical idea of "zero marginal rate of substitution" from economics, cf. also Duffie and Skiadas (1994). Intuitively, $\widehat{p}$ is defined such that an investor cannot increase his expected (exponential) utility by diverting an infinitesimal amount of his capital into the option contract. By (3.12), (3.19), and (3.20) we can consider the "fair price" $\widehat{p}$ as the limit of the utility-indifference valuations for $B$ for vanishing risk-aversion or infinitesimal contract volume, respectively. Moreover, $\widehat{p}$ provides a (sharp) lower bound for $\pi(B ; \alpha), \alpha \in(0, \infty)$.

Our next aim is to characterize the utility-indifference hedging strategy $\psi$ and the indifference price $\pi$. To this end, we formulate a martingale criterion which is sufficient to identify both of them and 
which serves as a verification theorem in the sequel. It also leads in a natural way to the definition of a utility-indifference price process $\left(\pi_{t}\right)_{t \in[0, T]}$, and reveals further general properties of $\left(\pi_{t}\right)$ and $\psi$. Our starting point is the following proposition which provides sufficient criteria for the solution to the dual problem (2.8). Basically, it is a reformulation of results by Grandits and Rheinländer (2002) which includes a terminal liability $B$; see the appendix for technical details.

Proposition 3.5 Assume (2.4). Suppose the density of $\bar{Q} \in \mathbb{P}_{a}$ takes the form

$$
\frac{d \bar{Q}}{d P}=\exp \left(-\alpha\left(\bar{c}+\int_{0}^{T} \bar{\vartheta} d S-B\right)\right) \quad \text { for some } \bar{c} \in \mathbb{R} \text { and } \bar{\vartheta} \in L(S)
$$

and one of the following two conditions holds:

(i) $\bar{\vartheta} \cdot S$ is a $\bar{Q}-\mathcal{B M O}$-martingale and $e^{\alpha\left(B+\varepsilon \int_{0}^{T} \bar{\vartheta} d S\right)} \in L^{1}(P)$ for some $\varepsilon>0$, or

(ii) $\bar{Q} \in \mathbb{P}_{f}$ and $\bar{\vartheta} \in \Theta_{\mathcal{M}}$.

Then $\bar{Q} \in \mathbb{P}_{e} \cap \mathbb{P}_{f}$ solves the dual problem i.e. $\bar{Q}=Q^{B}, \bar{\vartheta}=\vartheta^{B}$ and $\bar{c}=c^{B}$ (cf. Prop. 2.2).

As a consequence from variant (ii), one can now characterize the indifference price $\pi$ and the hedging strategy $\psi$ by a condition that is both necessary and sufficient:

Corollary 3.6 Suppose the assumptions for Proposition 2.2 hold.

1. The hedging strategy $\psi(B)$ is in $\Theta_{\mathcal{M}}$ and satisfies $\frac{d Q^{B}}{d Q^{0}}=\exp \left(-\alpha\left(\pi(B)+\int_{0}^{T} \psi(B) d S-B\right)\right)$.

2. Conversely: Let $\bar{\psi} \in \Theta_{\mathcal{M}}, \bar{\pi} \in \mathbb{R}$ and $\bar{Q} \in \mathbb{P}_{f}$ such that

$$
\frac{d \bar{Q}}{d Q^{0}}=\exp \left(-\alpha\left(\bar{\pi}+\int_{0}^{T} \bar{\psi} d S-B\right)\right)
$$

holds. Then we have $\psi(B)=\bar{\psi}, \pi(B)=\bar{\pi}$ and $Q^{B}=\bar{Q}$.

Remark 3.7 Part 2 of Corollary 3.6 implies in particular that the replicating strategy for a bounded attainable claim $B$ is the hedging strategy, as it should be. To see this, let $B=b+\int_{0}^{T} \bar{\psi} d S$ with $b \in \mathbb{R}$ and $\int \bar{\psi} d S$ bounded (uniformly in $t$ ). Taking $\bar{Q}:=Q^{0}$ and $\bar{\pi}:=b$, Part 2 of Corollary 3.6 yields that $\pi(B)=\bar{\pi}$ and $\psi(B)=\bar{\psi}$; that is, the indifference price equals the initial capital that is needed for the replication of $B$ and the hedging strategy is the replicating strategy.

\section{Proof of Corollary 3.6:}

1. By Proposition 2.2 we have $u\left(c^{B}-B\right)=-1=u\left(c^{0}-0\right)$, and we conclude that $\pi(B)=c^{B}-c^{0}$. Moreover, the hedging strategy $\psi(B)=\vartheta^{B}-\vartheta^{0}$ is in $\Theta_{\mathcal{M}}$. Computing the density of $d Q^{B}$ with respect to $d Q^{0}$ via $\frac{d Q^{B}}{d P}\left(\frac{d Q^{0}}{d P}\right)^{-1}$, again using Prop. 2.2, yields part 1 .

2. For the "sufficiency"-part, consider

$$
\frac{d \bar{Q}}{d P}=\left(\frac{d Q^{0}}{d P}\right) \frac{d \bar{Q}}{d Q^{0}}=\exp \left(-\alpha\left(\left(c^{0}+\bar{\pi}\right)+\int_{0}^{T}\left(\vartheta^{0}+\bar{\psi}\right) d S-B\right)\right) .
$$

Proposition 3.5-(ii) then yields $Q^{B}=\bar{Q}$, and $\vartheta^{B}=\vartheta^{0}+\bar{\psi}$ and $c^{B}=c^{0}+\bar{\pi}$ follow via Proposition 2.2. As in part $1, \pi(B)=c^{B}-c^{0}=\bar{\pi}$ holds, and $\psi(B)=\vartheta^{B}-\vartheta^{0}=\bar{\psi}$ by the definition of $\psi(B)$. 
Corollary 3.6 yields that the density process of $Q^{B}$ with respect to $Q^{0}$ takes the form

$$
\left.\frac{d Q^{B}}{d Q^{0}}\right|_{\mathcal{F}_{t}}=\exp \left(-\alpha\left(\pi(B)+\int_{0}^{t} \psi(B) d S-\pi_{t}\right)\right), \quad t \in[0, T]
$$

with $\left(\pi_{t}\right)_{t \in[0, T]}$ given by

$$
\pi_{t}=\frac{1}{\alpha} \log E_{Q^{0}}\left[\exp \left(\alpha\left(B-\int_{t}^{T} \psi(B) d S\right)\right) \mid \mathcal{F}_{t}\right], \quad t \in[0, T] .
$$

Note that $\left(\pi_{t}\right)_{t \in[0, T]}$ is a semimartingale with $\pi_{T}=B$ and $\pi_{0}=\pi(B)$. Moreover, we have

$$
E_{Q^{0}}\left[B \mid \mathcal{F}_{t}\right] \leq \pi_{t} \leq E_{Q^{B}}\left[B \mid \mathcal{F}_{t}\right], \quad t \in[0, T] .
$$

To see this, let $Z$ denote the density process of $Q^{B}$ with respect to $Q^{0}$. Starting from the inequalities $E_{Q^{0}}\left[\frac{Z_{T}}{Z_{t}} \log \frac{Z_{T}}{Z_{t}} \mid \mathcal{F}_{t}\right] \geq 0$ and $E_{Q^{B}}\left[\frac{Z_{T}^{-1}}{Z_{t}^{-1}} \log \frac{Z_{T}^{-1}}{Z_{t}^{-1}} \mid \mathcal{F}_{t}\right] \geq 0$, we can compute the conditional expectations, using the representation (3.23) for $Z$ and the fact that $\psi$ is in $\Theta_{\mathcal{M}}$. This yields (3.25).

Definition 3.8 Suppose the assumptions for Proposition 2.2 hold. We call the process $\left(\pi_{t}\right)_{t \in[0, T]}$ in the representation (3.23) the utility-indifference price process of $B$.

We will give a justification for this definition in the sequel. Beforehand, let us state a density process version of Corollary 3.6. An analogous reasoning leads to a density process version of Proposition 3.5 and thereby offers an economic interpretation for the indifference price process $\left(\pi_{t}\right)_{t \in[0, T]}$, see the remarks at the end of this section.

Corollary 3.9 Suppose the assumptions for Proposition 2.2 hold and we have

$$
\left.\frac{d \bar{Q}}{d Q^{0}}\right|_{\mathcal{F}_{t}}=\exp \left(-\alpha\left(\bar{\pi}_{0}+\int_{0}^{t} \bar{\psi} d S-\bar{\pi}_{t}\right)\right), \quad t \in[0, T]
$$

for some $\bar{Q} \in \mathbb{P}_{f}, \bar{\psi} \in \Theta_{\mathcal{M}}$, and a semimartingale $\left(\bar{\pi}_{t}\right)_{t \in[0, T]}$ with $\bar{\pi}_{T}=B$. Then $\psi(B)=\bar{\psi}$, $\pi(B)=\bar{\pi}_{0}, Q^{B}=\bar{Q}$ and $\left(\bar{\pi}_{t}\right)$ is the indifference price process.

Proof: Taking $t=T$, we obtain by part 2 of Corollary 3.6 that $\psi(B)=\bar{\psi}, \pi(B)=\bar{\pi}_{0}$ and $Q^{B}=\bar{Q}$. Comparing (3.23) and (3.26) then implies that $\left(\bar{\pi}_{t}\right)$ is the utility-indifference price process.

As an application of Corollary 3.9, we now show that the exponential indifference pricing exhibits an iterativity property. Such a property is already known in a static framework, cf. Gerber (1979), but it also holds in a dynamical market setting. Moreover, a corresponding iterativity also holds for the hedging strategy. However, the notion of iterativity must be reformulated to take into account the information flow $\mathbb{F}$ for a simple reason: In financial markets it matters at what time new information becomes available.

Corollary 3.10 (iterativity of $\pi$ and $\psi$ )

Suppose the assumptions for Proposition 2.2 hold and B is bounded. Let $\tau$ be a stopping time. Then the indifference price process and hedging strategy for the claim $\tilde{B}:=\pi_{\tau}(B)$ are given by $\pi_{t}(\tilde{B})=\pi_{t \wedge \tau}(B)$ and $\psi_{t}(\tilde{B})=\psi_{t}(B) 1_{\llbracket 0, \tau \rrbracket}(t)$, respectively. 
It is most natural to consider the claim $\tilde{B}$ as payable at time $\tau$. Note however that we are working throughout with discounted quantities, and in such a framework it does not really matter at which time payoffs are due.

Proof: Let $d \bar{Q}:=\left.\frac{d Q^{B}}{d Q^{0}}\right|_{\mathcal{F}_{\tau}} d Q^{0}$. From the representation (3.23) we have

$$
\left.\frac{d \bar{Q}}{d Q^{0}}\right|_{\mathcal{F}_{t}}=\exp \left(-\alpha\left(\pi_{0}(B)+\int_{0}^{t} \psi(B) 1_{\llbracket 0, \tau \rrbracket} d S-\pi_{t \wedge \tau}\right)\right), \quad t \in[0, T],
$$

with $\left\|\pi_{\tau}(B)\right\|_{\infty} \leq\|B\|_{\infty}<\infty$ by (3.25) and hypothesis. By Corollary 3.9 this yields the claim.

We conclude this section by giving an interpretation for the process $\left(\pi_{t}\right)$. To this end, suppose that (2.4) holds and that there are some $\bar{Q} \in \mathbb{P}_{a}, \bar{\vartheta} \in L(S)$ and a semimartingale $\left(c_{t}^{B}\right)_{t \in[0, T]}$ such that $c_{T}^{B}=B, \bar{\vartheta} \cdot S$ is a $\bar{Q}-\mathcal{B} \mathcal{M O}$-martingale,

$$
\left.\frac{d \bar{Q}}{d P}\right|_{\mathcal{F}_{t}}=\exp \left(-\alpha\left(c_{0}^{B}+\int_{0}^{t} \bar{\vartheta} d S-c_{t}^{B}\right)\right), \quad t \in[0, T]
$$

holds, and $e^{\alpha\left(B+\varepsilon \int_{0}^{T} \bar{\vartheta} d S\right)}$ is in $L^{1}(P)$ for some $\varepsilon>0$. Via Proposition 3.5 , this yields $\bar{Q}=Q^{B}$ and $\bar{\vartheta}=\vartheta^{B}$ and $c_{0}^{B}=c^{B}$, that is, we have the solution to the dual problem in Proposition 2.2. Since the terminal density determines the density process, we find that the process $\left(c_{t}^{B}\right)_{t \in[0, T]}$ is determined by $\vartheta^{B}$ and $B$. This dependence is analogous to the one in (3.24) and given by the formula

$$
c_{t}^{B}=\frac{1}{\alpha} \log E_{P}\left[\exp \left(\alpha\left(B-\int_{t}^{T} \vartheta^{B} d S\right)\right) \mid \mathcal{F}_{t}\right], \quad t \in[0, T] .
$$

Note that the expectation here is under the objective probability measure $P$ and not under $Q^{0}$ as in (3.24). This provides an interpretation to the processes $\left(c_{t}^{B}\right)$ and thereby also for $\left(\pi_{t}\right)$ : The investor who maximizes his $P$-expected utility under terminal liability $B$ follows his optimal trading strategy $\vartheta^{B}$. At time $t \in[0, T]$, he then faces the effective liability $B-\int_{t}^{T} \vartheta^{B} d S$ which is the difference between $B$ and the gains from trade that he is going to realize in the remaining time $(t, T]$. Note that the effective liability can be positive as well as negative. In the latter case, the investor enjoys an effective gain from present time $t$ until $T$. By (3.28), we can interpret $c_{t}^{B}$ as the current (exponential) $P$-certainty equivalent of this remaining effective liability given the information at time $t$ : At time $t, c_{t}^{B}$ is the "time- $t$-certain" liability that an investor would rate as good as the remaining effective liability in terms of expected exponential utility. For the investor with no liability the corresponding certainty equivalent is simply $c_{t}^{0}=\frac{1}{\alpha} \log E_{P}\left[\exp \left(\alpha\left(-\int_{t}^{T} \vartheta^{0} d S\right)\right) \mid \mathcal{F}_{t}\right]$, $t \in[0, T]$. We can calculate the density process $d Q^{B} /\left.d Q^{0}\right|_{\mathcal{F}_{t}}=d Q^{B} /\left.d P\right|_{\mathcal{F}_{t}} d Q^{0} /\left.d P\right|_{\mathcal{F}_{t}} ^{-1}, t \in[0, T]$, by using formula (3.27) (with $\bar{Q}=Q^{B}$ and $\bar{\vartheta}=\vartheta^{B}$ ). Substituting $\psi(B)=\vartheta^{B}-\vartheta^{0}$ and comparing the result with (3.26) then yields

$$
\pi_{t}=c_{t}^{B}-c_{t}^{0}, \quad t \in[0, T] .
$$


Hence, the current utility-indifference price is the difference of the current certainty equivalents of the effective liabilities between the investor with terminal liability $B$ and the ordinary investor who has no terminal liability. For further details about certainly-equivalence aspects, we refer to Frittelli (2000a) who defines a market-consistent economic value of a random financial position starting from a certainty-equivalence argument. For exponential utility preferences, this leads to the same valuation formula as the utility-indifference approach. In a Brownian framework, Rouge and El Karoui (2000) define a process $x_{t}^{C}$ (in their Section 4.2) as an essential supremum of certain conditional expectations over a set of martingale measures. One could show that their $x_{t}^{C}$ coincides with our process $c_{t}^{B}$.

\section{Results in semi-complete product models}

This section aims for more constructive results on the utility-indifference valuation and hedging approach, and also explores and illustrates its properties in more detail. To this end, we impose further structural assumptions on our financial market model, assuming a semi-complete product model which consists of a complete financial sub-market and additional independent sources of risk.

\subsection{The semi-complete product model}

We now specify the semi-complete model and the technical assumptions which are supposed to hold throughout the rest of Section 4. Let $\mathbb{F}^{0}=\left(\mathcal{F}_{t}^{0}\right)_{t \in[0, T]}$ and $\mathbb{I}^{i}=\left(\mathcal{I}_{t}^{i}\right)_{t \in[0, T]}, i \in \mathbb{N}$, be filtrations which satisfy the usual conditions of completeness and right-continuity, and have trivial $\sigma$-fields at $t=0$. We suppose that $S$ is adapted and locally bounded with respect to $\mathbb{F}^{0}$ and that

$$
\mathcal{F}_{T}^{0}, \mathcal{I}_{T}^{1}, \mathcal{I}_{T}^{2}, \ldots \quad \text { are independent under } P .
$$

Let $\mathbb{I}=\left(\mathcal{I}_{t}\right)$ be given by $\mathcal{I}_{t}:=\bigvee_{i=1}^{\infty} \mathcal{I}_{t}^{i}$ and define $\mathbb{F}=\left(\mathcal{F}_{t}\right)_{t \in[0, T]}$ via

$$
\mathcal{F}_{t}:=\mathcal{F}_{t}^{0} \vee \mathcal{I}_{t}=\mathcal{F}_{t}^{0} \vee \bigvee_{i=1}^{\infty} \mathcal{I}_{t}^{i}, \quad t \in[0, T]
$$

We assume that there is a unique $Q^{*}$ which is in $\mathbb{P}_{e} \cap \mathbb{P}_{f}$ and has an $\mathcal{F}_{T}^{0}$-measurable density, i.e.

$$
\left\{Q \in \mathbb{P}_{e} \mid Q \in \mathbb{P}_{f}, \frac{d Q}{d P} \text { is } \mathcal{F}_{T}^{0} \text {-measurable }\right\}=\left\{Q^{*}\right\}
$$

Hence, $S$ has the strong predictable representation property with respect to $\left(\mathbb{F}^{0}, Q^{*}\right)$ (see Jacod (1979), Cor. 11.4, and Kabanov and Stricker (2001), Cor. 1.3) and conditions (2.1) and (2.2) hold. Because of (4.3), condition (4.1) is equivalent to assuming that

$$
\mathcal{F}_{T}^{0}, \mathcal{I}_{T}^{1}, \mathcal{I}_{T}^{2}, \ldots \quad \text { are independent under } Q^{*}
$$

and it is easy to see that $Q^{*}=P$ on $\mathcal{I}_{T}$. By Lemma A.2, both $\mathbb{F}$ and $\mathbb{I}$ inherit the usual conditions from their sub-filtrations thanks to the independence assumption (4.1). 
Remark 4.1 Assumptions (4.3) and (4.1) mean that our model is composed of a complete financial market $\left(S, \mathbb{F}^{0}\right)$ - e.g. the Black-Scholes model or the Binomial-tree model - and additional independent sources (factors) $\mathbb{I}^{1}, \mathbb{I}^{2}, \ldots$ of risk which are independent from $\left(S, \mathbb{F}^{0}\right)$ under the objective probability $P$. Generic examples in the sequel are given by $P$-independent processes $S, Y^{1}, Y^{2}, \ldots$, with $\mathbb{F}^{0}:=\mathbb{F}^{S}$ and $\mathbb{I}^{i}:=\mathbb{F}^{Y^{i}}$ being the smallest right-continuous and complete filtrations generated by $S$ and $Y^{i}$, respectively. This class of models includes, for instance, the ones considered in Davis (2000), Henderson and Hobson (2002), Henderson (2002), and Young and Zariphopoulou (2002).

\subsection{The structure of the equivalent martingale measures}

For the sequel, we need some structural results on the solution $Q^{B}$ for the dual problem (2.8) and on the set $\mathbb{P}_{e}$ of equivalent martingale measures. Since $d Q^{*}=d Q /\left.d P\right|_{\mathcal{F}_{T}^{0}} d P$ holds for any $Q \in \mathbb{P}_{f}$ we have $H\left(Q^{*} \mid P\right) \leq H(Q \mid P)$ for $Q \in \mathbb{P}_{f}$, i.e. $Q^{*}$ is the minimal entropy martingale measure:

$$
Q^{*}=Q^{0}
$$

A similar reasoning yields

Lemma 4.2 Suppose $B$ is $\mathcal{F}_{T}^{0} \vee \mathcal{I}_{T}^{k}$-measurable for some $k$ and satisfies (2.4). Then the density $d Q^{B} / d Q^{*}$ of the optimal measure $Q^{B}$ for the dual problem (2.8) is $\mathcal{F}_{T}^{0} \vee \mathcal{I}_{T}^{k}$-measurable.

Proof: We may assume that $S$ is bounded, otherwise one could $\mathbb{F}^{0}$-localize so that it is. It suffices to consider the case $k=1$. Under the present conditions, the optimal measure $Q^{B}$ to (2.8) exists (see the proof of Proposition 2.2). Define $d Q^{\prime}:=d Q^{B} /\left.d P_{B}\right|_{\mathcal{F}_{T}^{0} \vee \mathcal{I}_{T}^{1}} d P_{B}$ and recall that $d P_{B}:=\left(E\left[e^{\alpha B}\right]\right)^{-1} e^{\alpha B} d P$. Then $S$ is a martingale with respect to $\left(Q^{\prime},\left(\mathcal{F}_{t}^{0} \vee \mathcal{I}_{t}^{1}\right)_{t \in[0, T]}\right)$ and

$$
\frac{d Q^{\prime}}{d Q^{*}}=\left(\frac{d Q^{\prime}}{d P_{B}}\right)\left(\frac{d P_{B}}{d P}\right)\left(\frac{d P}{d Q^{*}}\right) \quad \text { is } \mathcal{F}_{T}^{0} \vee \mathcal{I}_{T}^{1} \text {-measurable. }
$$

In combination with (4.4), this implies that $\mathcal{F}_{T}^{0} \vee \mathcal{I}_{T}^{1}$ is $Q^{\prime}$-independent from $\mathcal{I}_{T}^{2} \vee \mathcal{I}_{T}^{3} \vee \ldots$, and so $S$ is also a $\left(Q^{\prime}, \mathbb{F}\right)$-martingale. By construction of $Q^{\prime}$ we have $H\left(Q^{\prime} \mid P_{B}\right) \leq H\left(Q^{B} \mid P_{B}\right)$ and $Q^{\prime} \sim P$. Since $Q^{B}$ is unique and minimizes $H\left(Q \mid P_{B}\right)$ over $Q \in \mathbb{P}_{a}$ (cf. Lemma A.1), this yields $Q^{\prime}=Q^{B}$.

In the semi-complete model, the set $\mathbb{P}_{e}$ of equivalent martingale measures has a rather simple structure which is analyzed next. We denote by $E^{*}$ the expectation with respect to $Q^{*}$.

Lemma 4.3 Let $Q$ be some measure equivalent to $P$ and let $Z$ denote the $\mathbb{F}$-density process of $Q$ with respect to $Q^{*}$. Then $Q$ is an element of $\mathbb{P}_{e}$ if and only if

$$
E^{*}\left[Z_{t} \mid \mathcal{F}_{t}^{0} \vee \mathcal{I}_{s}\right]=Z_{s} \quad \text { for all } \quad 0 \leq s \leq t \leq T .
$$

In particular, taking $s=0$ gives $E^{*}\left[Z_{t} \mid \mathcal{F}_{t}^{0}\right]=1$ for $t \in[0, T]$. 
Proof: Again, we can assume that $S$ is bounded. Otherwise we can $\mathbb{F}^{0}$-localize appropriately. Let us first show necessity: Fix $s \leq T$ and define

$$
\widetilde{Z}_{t}:=E^{*}\left[Z_{T} \mid \mathcal{F}_{t}^{0} \vee \mathcal{I}_{s}\right]=E^{*}\left[Z_{t} \mid \mathcal{F}_{t}^{0} \vee \mathcal{I}_{s}\right], \quad t \in[s, T]
$$

where the equality follows via conditioning on $\mathcal{F}_{t}$. To show $(4.6)$, it suffices to prove

$$
\widetilde{Z}_{t}=\widetilde{Z}_{s} \quad \text { for } \quad t \in[s, T]
$$

To this end, define $\left(L_{t}\right)_{t \in[0, T]}$ by $L_{t}:=1_{[0, s)}(t)+\left(\widetilde{Z}_{t} / \widetilde{Z}_{s}\right) 1_{[s, T]}(t)$. Then

$$
S \text { and } L \text { are martingales with respect to }\left(Q^{*},\left(\mathcal{F}_{t}^{0} \vee \mathcal{I}_{s}\right)_{t \in[0, T]}\right)
$$

by the independence hypothesis (4.4) and the construction of $L$, respectively. A little calculation shows that also $L S$ is a $\left(Q^{*},\left(\mathcal{F}_{t}^{0} \vee \mathcal{I}_{s}\right)_{t \in[0, T]}\right)$-martingale. In fact,

$$
Z_{u} S_{u}=E^{*}\left[Z_{T} S_{t} \mid \mathcal{F}_{u}\right]=E^{*}\left[E^{*}\left[Z_{T} \mid \mathcal{F}_{t}^{0} \vee \mathcal{I}_{u}\right] S_{t} \mid \mathcal{F}_{u}\right], \quad s \leq u \leq t \leq T,
$$

implies - by taking $E^{*}\left[\cdot \mid \mathcal{F}_{u}^{0} \vee \mathcal{I}_{s}\right]$ of both sides - that

$$
E^{*}\left[Z_{u} \mid \mathcal{F}_{u}^{0} \vee \mathcal{I}_{s}\right] S_{u}=E^{*}\left[E^{*}\left[Z_{T} \mid \mathcal{F}_{t}^{0} \vee \mathcal{I}_{u}\right] S_{t} \mid \mathcal{F}_{u}^{0} \vee \mathcal{I}_{s}\right], \quad s \leq u \leq t \leq T
$$

Via conditioning on $\mathcal{F}_{t}^{0} \vee \mathcal{I}_{s}$, one sees that the right hand side equals $E^{*}\left[E^{*}\left[Z_{T} \mid \mathcal{F}_{t}^{0} \vee \mathcal{I}_{s}\right] S_{t} \mid \mathcal{F}_{u}^{0} \vee \mathcal{I}_{s}\right]$. Using the definitions of $\widetilde{Z}$ and $L$, we can then rewrite (4.9) as

$$
L_{u} S_{u}=E^{*}\left[L_{t} S_{t} \mid \mathcal{F}_{u}^{0} \vee \mathcal{I}_{s}\right] \quad \text { for } \quad s \leq u \leq t \leq T .
$$

In combination with the definition of $L$ and (4.8), this establishes that $L S$ is a martingale with respect to $\left(Q^{*},\left(\mathcal{F}_{t}^{0} \vee \mathcal{I}_{s}\right)_{t \in[0, T]}\right)$. From (4.4) and (4.3) it follows by Theorem 3.2 in Amendinger et al. (2003) that $S$ has the strong predictable representation property with respect to $\left(Q^{*},\left(\mathcal{F}_{t}^{0} \vee\right.\right.$ $\left.\left.\left.\mathcal{I}_{s}\right)\right)_{t \in[0, T]}\right)$. That is, any local $\left(Q^{*},\left(\mathcal{F}_{t}^{0} \vee \mathcal{I}_{s}\right)_{t \in[0, T]}\right)$-martingale orthogonal to $S$ must be constant (Jacod (1979), Theorem 11.3). Hence $L$ is constant at $L_{0}=1$ and this establishes (4.7).

Finally, to show sufficiency just note that (4.6) and (4.4) imply

$$
E^{*}\left[Z_{t} S_{t} \mid \mathcal{F}_{s}^{0} \vee \mathcal{I}_{s}\right]=E^{*}\left[S_{t} E^{*}\left[Z_{t} \mid \mathcal{F}_{t}^{0} \vee \mathcal{I}_{s}\right] \mid \mathcal{F}_{s}^{0} \vee \mathcal{I}_{s}\right]=E^{*}\left[S_{t} Z_{s} \mid \mathcal{F}_{s}^{0} \vee \mathcal{I}_{s}\right]=Z_{s} S_{s}
$$

\subsection{A computation scheme and explicit bounds}

We now derive a backward computation scheme for the utility-indifference price process in the case where the additional information flow $\mathbb{I}=\left(\mathcal{I}_{t}\right)_{t \in[0, T]}$ is piecewise constant, i.e.

$$
\mathcal{I}_{t}=\mathcal{I}_{t_{k}} \quad \text { for } t \in\left[t_{k}, t_{k+1}\right) \text { and } k=0, \ldots, n-1 \text {, }
$$

with deterministic times $0=t_{0}<t_{1}<\cdots<t_{n}=T$. This will also lead to simple upper and lower bounds for the utility-indifference price which are valid even if condition (4.11) does not hold. 
Theorem 4.4 (Computation scheme) Assume (2.4), (2.5), and (4.11). Then the utility-indifference price process $\left(\pi_{t}\right)$ is recursively determined by $\pi_{t_{n}}=B$ and

$$
\pi_{t}=E^{*}\left[\frac{1}{\alpha} \log E_{P}\left[e^{\alpha \pi_{t_{k+1}}} \mid \mathcal{F}_{t_{k+1}}^{0} \vee \mathcal{I}_{t_{k}}\right] \mid \mathcal{F}_{t}^{0} \vee \mathcal{I}_{t_{k}}\right] \quad, t \in\left[t_{k}, t_{k+1}\right)
$$

and the utility-indifference price at time $t=0$ is $\pi(B ; \alpha)=\pi_{0}$.

Having this result, a second reading of the proof reveals (via (4.14)) that

$$
\pi_{t}=\pi_{t_{k}}+\int_{t_{k}}^{t} \psi(B) d S, \quad t \in\left[t_{k}, t_{k+1}\right)
$$

That means, the indifference price process $\left(\pi_{t}\right)$ is piecewise self-financing, and the hedging strategy $\psi(B)$ is the replicating strategy for $\frac{1}{\alpha} \log E_{P}\left[\exp \left(\alpha \pi_{t_{k+1}}\right) \mid \mathcal{F}_{t_{k+1}}^{0} \vee \mathcal{I}_{t_{k}}\right]$ over the time period $\left(t_{k}, t_{k+1}\right]$. As the subsequent proof reveals, one could alternatively state (4.12) with the expectation $E_{P}$ replaced by $E^{*}$. However, the given formula emphasizes where the true world probability $P$ comes into play, and is also more reminiscent to the heuristics in Remark 4.5.

In a way, the computation scheme for the utility-indifference price resembles the common backwards computation method of the replication price in the binomial tree model: The indifference price at time $t_{k}$ can be computed as the conditional expectation of a functional of the price at time $t_{k+1}$. In fact, the scheme reduces to the unique no-arbitrage valuation $\pi_{t}=E^{*}\left[B \mid \mathcal{F}_{t}\right]$ when the claim only depends on tradable factors of risk, that is when $B$ is $\mathcal{F}_{T}^{0}$-measurable. We emphasize that the measures $Q^{*}$ and $P$ are typically well-known - in contrast to $Q^{B}$. Note that the computation of $\left(\pi_{t}\right)$ is "local" in time, although $\pi$ is derived from an optimization problem whose solution is typically not myopic (i.e. optimizing exponential utility locally seperately over each time period does in general not lead to an investment strategy which maximizes exponential utility from terminal wealth over all periods up to time $T$, as it would be the case for logarithmic utility, cf. e.g. Becherer (2001a)). The scheme provides an explicit recursion formula and does not necessitate a numerical (approximative) search for the intermediate optimization at each period, as it appears often in dynamical programming.

Remark 4.5 1. Before proving Theorem 4.4, we give a heuristic explanation for the recursion formula (4.12). At time $t_{k+1}$ the utility-indifference price of the claim $B$ for an investor with exponential utility is $\pi_{t_{k+1}}$ and the investor possesses the information $\mathcal{F}_{t_{k+1}}^{0} \vee \mathcal{I}_{t_{k+1}}$. Before he comes to know the new additional information, he has information $\mathcal{F}_{t_{k+1}}^{0} \vee \mathcal{I}_{t_{k}}$ and would assign the certainty equivalent $\tilde{\pi}_{t_{k+1}}:=\frac{1}{\alpha} \log E_{P}\left[\exp \left(\alpha \pi_{t_{k+1}}\right) \mid \mathcal{F}_{t_{k+1}}^{0} \vee \mathcal{I}_{t_{k}}\right]$ to $\pi_{t_{k+1}}$. By no-arbitrage considerations, the only reasonable price for $\tilde{\pi}_{t_{k+1}}$ at time $t \in\left[t_{k}, t_{k+1}\right)$ is $\pi_{t}=E^{*}\left[\tilde{\pi}_{t_{k+1}} \mid \mathcal{F}_{t}\right]$ since $\tilde{\pi}_{t_{k+1}}$ can be replicated from $\pi_{t}$ by a self-financing trading strategy. This replication is possible because the model is "piecewise complete". More precisely, Theorem 3.2 in Amendinger et al. (2003) shows that $\left(S_{t}\right)_{t \in\left[t_{k}, t_{k+1}\right]}$ has the representation property with respect to $\left(Q^{*},\left(\mathcal{F}_{t}^{0} \vee \mathcal{I}_{t_{k}}\right)_{t \in\left[t_{k}, t_{k+1}\right]}\right)$.

2. Independently, Musiela and Zariphopoulou (2002b) have obtained a different proof for a formula 
like (4.12) by direct calculations for the primal utility optimization problem in a one-period model. $\diamond$

Proof of Theorem 4.4: Corollary 3.6 and the subsequent remarks show that the $\mathbb{F}$-density process of $Q^{B}$ with respect to $Q^{0}$ has the form

$$
Z_{t}=\exp \left(-\alpha\left(\pi_{0}+\int_{0}^{t} \psi(B) d S-\pi_{t}\right)\right), \quad t \in[0, T]
$$

with $\pi_{T}=B$ and $\psi(B) \in \Theta_{\mathcal{M}}$. For $t \in\left[t_{k}, t_{k+1}\right)$ we obtain

$$
\begin{aligned}
1 & =E^{*}\left[\frac{Z_{t_{k+1}}}{Z_{t}} \mid \mathcal{F}_{t_{k+1}}^{0} \vee \mathcal{I}_{t}\right] \\
& =\exp \left(-\alpha\left(\int_{\left(t, t_{k+1}\right]} \psi(B) d S+\pi_{t}\right)\right) E^{*}\left[e^{\alpha \pi_{t_{k+1}}} \mid \mathcal{F}_{t_{k+1}}^{0} \vee \mathcal{I}_{t_{k}}\right]
\end{aligned}
$$

using Lemma 4.3, assumption (4.11) and the fact that $\int_{\left(t, t_{k+1}\right]} \psi(B) d S$ is $\mathcal{F}_{t_{k+1}}^{0} \vee \mathcal{I}_{t_{k}}$-measurable due to (4.11) and the predictability of $\psi$. This measurability is readily verified when the integrand is an elementary predictable process. By standard approximation arguments the measurability then also holds for left continuous processes with right limits, for bounded predictable processes, and finally for integrable predictable processes (cf. Mémin (1980), Lemma V.3). Solving for $\pi_{t}$ in (4.13) leads to

$$
\pi_{t}=-\int_{\left(t, t_{k+1}\right]} \psi(B) d S+\frac{1}{\alpha} \log E^{*}\left[e^{\alpha \pi_{t_{k+1}}} \mid \mathcal{F}_{t_{k+1}}^{0} \vee \mathcal{I}_{t}\right], \quad t \in\left[t_{k}, t_{k+1}\right),
$$

and taking $Q^{*}$-expectations conditional on $\mathcal{F}_{t}^{0} \vee \mathcal{I}_{t}=\mathcal{F}_{t}^{0} \vee \mathcal{I}_{t_{k}}$ yields (4.12). To see this, note that $\psi(B) \cdot S$ is a $Q^{*}$-martingale, and $E^{*}\left[\exp \left(\alpha \pi_{t_{k+1}}\right) \mid \mathcal{F}_{t_{k+1}}^{0} \vee \mathcal{I}_{t_{k}}\right]$ equals $E_{P}\left[\exp \left(\alpha \pi_{t_{k+1}}\right) \mid \mathcal{F}_{t_{k+1}}^{0} \vee \mathcal{I}_{t_{k}}\right]$ by hypothesis (4.4).

To illustrate some qualitative properties of the indifference price $\pi$ and to clarify the computation method, we next give an example with a simple claim and model. The outlined ideas are extendable to more complex contingent claims. A more quantitative analysis shall be done elsewhere.

Example 4.6 Consider a claim $B$ whose payoff depends on the evolution of a tradable asset $S$ but takes place only if a certain additional event " $\tau$ " does not happen until maturity. Let $S$ evolve according to the binomial tree model, i.e.

$$
S_{(k+1) \frac{T}{n}}=S_{k \frac{T}{n}} Y_{k+1} \quad \text { for } k=0, \ldots, n-1,
$$

where $S_{0} \in(0, \infty), d=1$, and $Y_{1}, Y_{2}, \ldots$ are i.i.d. with $P\left[Y_{k}=\mathcal{U}\right]=1-P\left[Y_{k}=1 / \mathcal{U}\right]=: p \in(0,1)$ for some $\mathcal{U} \in(1, \infty)$. By piecewise constant interpolation this model can be embedded in our continuous-time framework, taking $S_{t}:=S_{k T / n}$ for $t \in\left[k \frac{T}{n},(k+1) \frac{T}{n}\right)$. In order to view $S$ as usual as an approximation of the Black-Scholes model, we parameterize $\mathcal{U}:=\exp (\sigma \sqrt{T / n})$ by a volatility parameter $\sigma \in(0, \infty)$. Additional non-tradable sources of risk are represented by a Poisson process 
$N, P$-independent of $S$, with intensity $\lambda>0$. Let $\widetilde{N}_{t}:=N_{k T / n}, t \in\left[k \frac{T}{n},(k+1) \frac{T}{n}\right)$, be an observation of $N$ in discrete time. The 'event' is taken to occur at the time $\tau:=\inf \left\{t: \widetilde{N}_{t}>0\right\}$ of the first jump of $\widetilde{N}_{t}$. With $\mathbb{F}^{0}:=\mathbb{F}^{S}$ and $\mathbb{I}:=\mathbb{F}^{\widetilde{N}}$, the example fits in the framework of Section 4.1. Note that $N, Y_{1}, Y_{2}, \ldots$ are $Q^{*}$-independent (cf. 4.4), $Q^{*}\left[Y_{k}=\mathcal{U}\right]=1-Q^{*}\left[Y_{k}=\mathcal{U}^{-1}\right]=: q=\frac{1-1 / \mathcal{U}}{\mathcal{U}-1 / \mathcal{U}}$ for all $k$, and the $Q^{*}$-intensity of $N$ is the same as under $P$ since $Q^{*}=P$ on $\mathcal{I}_{T}$.

Via Theorem 4.4 one could now value various claims whose payoffs depend on the joint evolution of $S$ and $\widetilde{N}$. Here, let us consider a claim

$$
B:=S_{T} 1_{\left\{N_{T}=0\right\}}=S_{T} 1_{\{\tau>T\}},
$$

that simply pays one unit of the tradable asset, say a stock index, provided that some further (insurance) event - technically the stopping time $\tau$ - has not happened until maturity $T$. The backwards calculation scheme of Theorem 4.4 is somewhat similar to the standard method for the pricing of (only) replicable options in the complete standard binomial model that does not contain non-tradable factors of risk. But a (conditional) 2-step expectation is needed now. The scheme (4.12) can be implemented in a tree model for $\left(S_{t}, \widetilde{N}_{t}\right)$ with a

$$
\text { double tree-step } \mathcal{F}_{t_{k}} \stackrel{\Delta S}{\longrightarrow} \mathcal{F}_{t_{k+1}}^{0} \vee \mathcal{I}_{t_{k}} \stackrel{\Delta \widetilde{N}}{\longrightarrow} \mathcal{F}_{t_{k+1}} \text { for each time step } t_{k} \rightarrow t_{k+1},
$$

one for each processes' increment. As usual, claims like $B$ can be treated efficiently in a recombinant tree (cf. Baxter and Rennie (1996), ch. 2.2) while payoffs that depend on the whole path would necessitate a non-recombinant tree. For the figures, we computed $\left(\pi_{t}\right)$ on a tree-grid with various starting values for $S_{0}$. To illustrate properties of the utility-indifference pricing, it is more instructive to plot some qualitative quantities instead of the utility-indifference prices themselves. Those quantities are plotted as a function of the tree-grid's coordinates and interpolated to a surface.

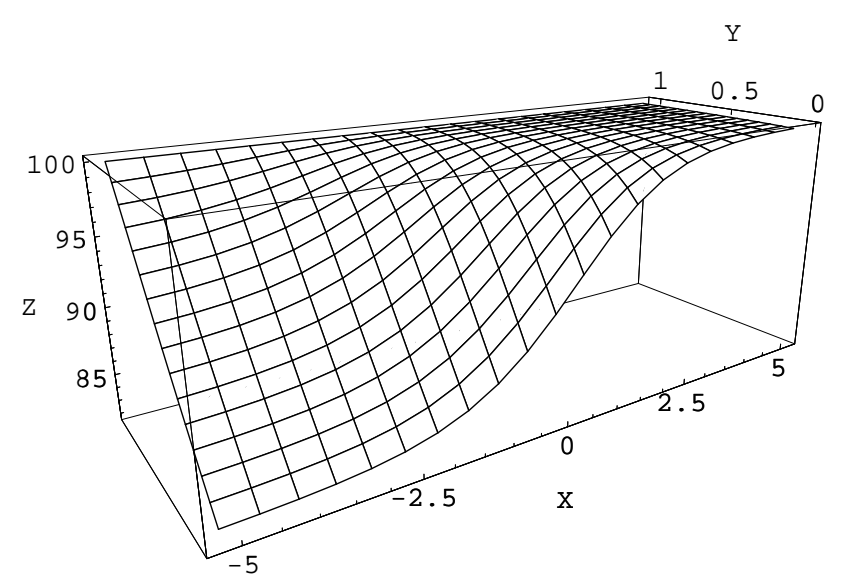

Figure 1: The utility-indifference price as a fraction of the superreplication price

[Quantity (4.15) ( $Z$-axis) as a function of $\log \left(S_{t} / S_{0}\right)(X$-axis $)$ and $t(Y$-axis $\left.)\right]$

As concrete parameters, let $T:=1, \sigma=1.0, n:=15, \lambda(t):=0.2$, and risk aversion $\alpha:=1$. This leads to $P\left[N_{T}=0\right] \approx 0.82, \mathcal{U} \approx 1.295, q \approx 0.483$, and for, say, $S_{0}:=1$ one obtains $\pi(B ; 1) \approx 0.894$. 
More generally, the indifference price process $\left(\pi_{t}\right)$ for $B$ is a function of $\left(t, S_{t}\right)$ on $\llbracket 0, \tau \llbracket$, and null on $\llbracket \tau, T \rrbracket$. Due to Kramkov (1996), we know that $\sup _{Q \in \mathbb{P}_{e}} E_{Q}[B]$ is the super-replication value, i.e. the smallest initial amount needed to eliminate (a.s.) the possibility of any losses from $B$ by dynamical trading. By (3.11) this amount equals $\lim _{\alpha \uparrow \infty} \pi(B ; \alpha)$ and can be interpreted as the utility-indifference price 'for infinite risk aversion'. Figure 1 shows the relation (in percent) of the indifference value to the super-replication price provided that $\widetilde{N}$ has not jumped so far, that is

$$
100 \frac{\pi_{t}}{\sup _{Q \in \mathbb{P}_{e}} E_{Q}\left[B \mid \mathcal{F}_{t}\right]}=100 \frac{\pi_{t}}{S_{t}} \quad \text { on } \llbracket 0, \tau \llbracket .
$$

In turn, for vanishing risk aversion $\alpha \downarrow 0$ the utility-indifference price tends to $E^{*}[B]$ by (3.12). Recalling that $Q^{*}=P$ on $\mathcal{I}_{T}$, the latter limit can be seen as a risk-neutral valuation (under $P$ ) with respect to non-tradable factors of risk. In this sense, the fraction in

$$
100 \frac{\pi_{t}-E^{*}\left[B \mid \mathcal{F}_{t}\right]}{E^{*}\left[B \mid \mathcal{F}_{t}\right]}=100 \frac{\pi_{t}-S_{t} P\left[\tau>T \mid \mathcal{F}_{t}\right]}{S_{t} P\left[\tau>T \mid \mathcal{F}_{t}\right]} \quad \text { on } \llbracket 0, \tau \llbracket .
$$

represents the relative safety loading of the indifference valuation $\pi_{t}(B ; 1)$ for risk aversion $\alpha=1$ with respect to the risk neutral valuation "for $\alpha=0$ ". Figure 2 shows this relative safety loading (in percent) as a function of $S_{t}$ and $t$, given that $\widetilde{N}$ has not jumped up to time $t$. Finally, let us

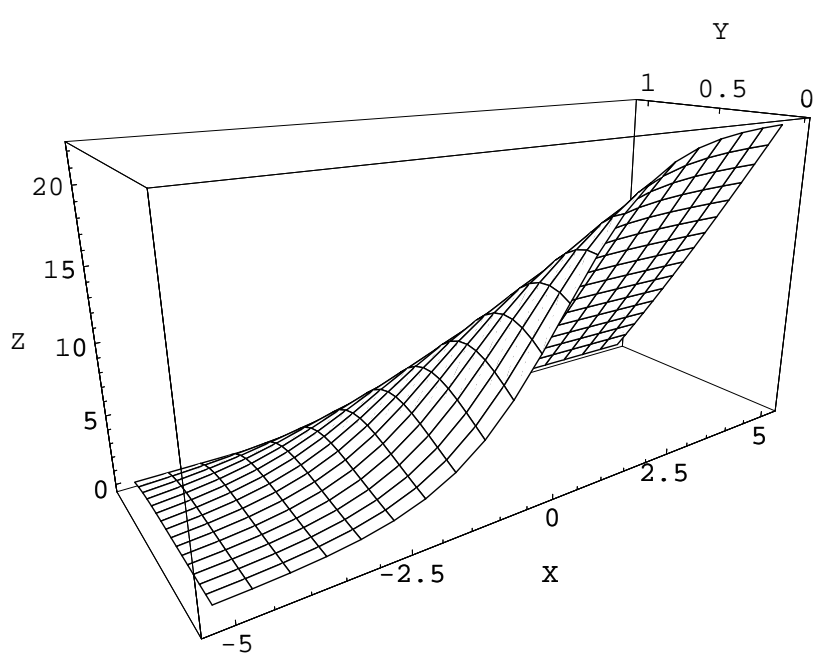

Figure 2: Relative safety loading of the utility-indifference price

[Quantity (4.16) (Z-axis) as a function of $\log \left(S_{t} / S_{0}\right)(X$-axis $)$ and $t(Y$-axis $\left.)\right]$

explain the phenomena showing up in Figures 1 and 2. When the claim is 'still alive', that is for $(t, \omega) \in \llbracket 0, \tau \llbracket$, the issuer faces the potential obligation to pay the liability $S_{T}$ at time $T$ in case $\tau>T$. The larger the current value of $S_{t}$ the larger is the potential liability $B=\left(S_{t}\left(S_{T} / S_{t}\right)\right) 1_{\{\tau>T\}}$ since $\left(S_{T} / S_{t}\right)$ is independent of $\mathcal{F}_{t}$. Hence, the potential liability becomes more challenging for the financial power of the issuer at higher values of $S_{t}$. Consequently, the safety loading incorporated in the risk averse valuation $\pi_{t}$ of the liability increases, and $\pi$ tends to the super-replication value in the extreme case; cf. the right sides of the figures. In contrast, for rather small values of $S_{t}$ the potential liability poses no serious problems to the issuer's abilities to take risk. Consequently, 
his valuation basically coincides with the risk neutral valuation which is the 'survival probability' $P[\tau>T \mid \tau>t]$ multiplied by the replication value $S_{t}$ for $S_{T}$. This is shown on the left sides of the figures. Intuitively plausible, the safety loading decreases as the time $T-t$ to maturity decreases because this reduces the probability for a still possible event at $\tau \in(t, T]$; i.e. the uncertainty about the non-tradable risk (from $\tau$ ) still present in the claim becomes smaller and the potential (effective) liability becomes more predictable. In contrast, the uncertainly about $S_{T}$ stems from a tradable source of risk and could be perfectly hedged at any time $t$ by trading in $S$ (simply buy and hold one unit of the asset for $S_{t}$ ) - if it would only be separate from the trigger event $\{\tau>T\}$. In a way, we have exhibited dependencies of the utility-indifference price on the size of a risky position which are similar as the results from Corollary 3.3. But here the effects concern the whole valuation process and vary over time, dynamically adapted to the current risk exposure of the issuer.

The next result shows that the more accurate and early the information on the non-marketed sources of risk (that is $\mathcal{I}_{T}$ ) comes in (via $\mathbb{I}$ ), the lower becomes the utility-indifference price.

Proposition 4.7 (Monotonicity of $\pi$ with respect to additional information)

Let $\check{\mathbb{I}}$ and $\widehat{\mathbb{I}}$ be filtrations satisfying the usual conditions, with $\check{\mathcal{I}}_{T}=\mathcal{I}_{T}=\widehat{\mathcal{I}}_{T}$ and such that

$$
\check{\mathcal{I}}_{t} \subseteq \mathcal{I}_{t} \subseteq \widehat{\mathcal{I}}_{t} \quad \text { holds for } t \in[0, T] .
$$

Let $\check{\mathbb{F}}:=\mathbb{F}^{0} \vee \check{\mathbb{I}}, \widehat{\mathbb{F}}:=\mathbb{F}^{0} \vee \widehat{\mathbb{I}}$, and recall $\mathbb{F}=\mathbb{F}^{0} \vee \mathbb{I}$ from (4.2). Suppose (2.4) holds and $\Theta(\mathbb{H}):=\Theta_{3}(\mathbb{H})$ for each $\mathbb{H} \in\{\check{\mathbb{F}}, \mathbb{F}, \widehat{\mathbb{F}}\}$. Then, the utility-indifference prices which correspond to the information flows $\check{\mathbb{F}}, \mathbb{F}$, and $\widehat{\mathbb{F}}$, respectively, satisfy the relations

$$
\pi(B ; \check{\mathbb{F}}) \geq \pi(B ; \mathbb{F}) \geq \pi(B ; \widehat{\mathbb{F}})
$$

Note that these bounds do not require assumption (4.11).

Let us comment on some technical aspects in the assumptions. For simplicity, our general framework assumes $\mathcal{F}_{T}=\mathcal{F}$ and we assume $\check{\mathcal{I}}_{T}=\mathcal{I}_{T}=\widehat{\mathcal{I}}_{T}$ just to stay in this setting and to have $\mathcal{H}_{T}=\mathcal{F}$ for all $\mathbb{H} \in\{\check{\mathbb{F}}, \mathbb{F}, \widehat{\mathbb{F}}\}$. To work in our general duality framework, we choose $\Theta(\mathbb{H})=\Theta_{3}(\mathbb{H})$ to have the duality (2.5) for each $\mathbb{H}$, cf. Example 2.1. Alternatively, one could impose the more general but more abstract assumption that (2.5) holds for each $\mathbb{H}$.

Proof: First, one encounters a minor technical problem since $\widehat{\mathcal{I}}_{0}$ and hence $\widehat{\mathcal{F}}_{0}$ might be not trivial (this is needed for Theorem 4.8). So the filtration $\widehat{\mathbb{F}}$ a priori does not fit in our framework which assumes a trivial initial filtration. But this problem can be overcome as follows: Consider the time interval $[-1, T]$ (instead of $[0, T]$ ), and let $S_{t}:=S_{0}$ for $t \in[-1,0)$ with all filtrations being trivial on $[-1,0)$. By this trick, we placed ourself within the framework for all filtrations $\check{\mathbb{F}}, \mathbb{F}$, and $\widehat{\mathbb{F}}$. The sets $\mathbb{P}_{f}$ of martingale measures corresponding to the different filtrations satisfy the inclusions

$$
\mathbb{P}_{f}(\check{\mathbb{F}}) \supseteq \mathbb{P}_{f}(\mathbb{F}) \supseteq \mathbb{P}_{f}(\widehat{\mathbb{F}})
$$


To see this, note that $S$ is locally bounded and adapted with respect to $\mathbb{F}^{0}, \mathbb{F}^{0}$ is a sub-filtration of $\check{\mathbb{F}}$, and $\check{\mathcal{F}}_{t} \subseteq \mathcal{F}_{t} \subseteq \widehat{\mathcal{F}}_{t}$ because of (4.17). This implies (4.19), since a martingale is also a martingale with respect to a smaller filtration, if it is adapted to the latter.

The independence of $\mathcal{F}_{T}^{0}$ and $\mathcal{I}_{T}$ under $Q^{*}$ implies that $Q^{*}$ is in $\mathbb{P}_{f}(\widehat{\mathbb{F}})$ and this yields $Q^{*} \in \mathbb{P}_{f}(\mathbb{H})$ for $\mathbb{H} \in\{\check{\mathbb{F}}, \mathbb{F}, \widehat{\mathbb{F}}\}$. For $\mathbb{H}=\check{\mathbb{F}}$, we have $H\left(Q^{*} \mid P\right) \leq H(Q \mid P)$ for any $Q \in \mathbb{P}_{f}(\check{\mathbb{F}})$ since $d Q^{*}=\left.\frac{d Q}{d P}\right|_{\mathcal{F}_{T}^{0}} d P$. By (4.19), this implies that $Q^{*}$ minimizes $H(Q \mid P)$ over $\mathbb{P}_{f}(\mathbb{H})$ for any $\mathbb{H} \in\{\check{\mathbb{F}}, \mathbb{F}, \widehat{\mathbb{F}}\}$, i.e.,

$$
Q^{0}(\mathbb{H})=Q^{*} \quad \text { for } \quad \mathbb{H} \in\{\check{\mathbb{F}}, \mathbb{F}, \widehat{\mathbb{F}}\} .
$$

From (4.19), (4.20) and (3.1) we finally conclude that the relations (4.18) hold.

To obtain simple bounds for the indifference price $\pi(B ; \alpha)$ in the next result, let us now consider the following extreme cases of additional information: On the one hand, there might be no additional information before terminal time $T$ such that $\mathcal{I}_{t}$ is trivial for $t \in[0, T)$. The opposite extreme is the case where all additional information is available from the beginning, that is $\mathcal{I}_{0}=\mathcal{I}_{T}$. Using the information-monotonicity of the utility-indifference price, the indifference prices which correspond to these extreme situations provide bounds for the utility-indifference price in an intermediate information stage. Due to Theorem 4.4, these bounds can be given in explicit form. To compute them, one just has to calculate two expectations, one of them conditional, under the measures $P$ and $Q^{*}$ which are typically well known - in contrast to $Q^{B}$. We note that Møller (2003b) obtained a similar result for an indifference approach that relies on quadratic objective functions (instead of exponential utility). A first comparison is made by Example 4.9.

Theorem 4.8 (Simple bounds) Assume (2.4) and (2.5). Then

$$
\frac{1}{\alpha} \log E_{P}\left[\exp \left(\alpha E^{*}\left[B \mid \mathcal{I}_{T}\right]\right)\right] \leq \pi(B ; \alpha) \leq E^{*}\left[\frac{1}{\alpha} \log E_{P}\left[\exp (\alpha B) \mid \mathcal{F}_{T}^{0}\right]\right] .
$$

Note that these bounds do not require assumption (4.11).

In the special cases where the claim $B$ either only depends on the complete sub-market or only depends on market-independent sources of risk, the upper and lower bounds in (4.21) coincide, and

$$
\pi(B ; \alpha)=\left\{\begin{array}{cl}
E^{*}[B] & \text { for } B \text { being } \mathcal{F}_{T}^{0} \text {-measurable } \\
\frac{1}{\alpha} \log E_{P}[\exp (\alpha B)] & \text { for } B \text { being } \mathcal{I}_{T} \text {-measurable }
\end{array}\right.
$$

Moreover, one can verify that the indifference hedging strategy $\psi(B)$ is the replicating strategy in the first case and constant at null in the second.

Proof of Theorem 4.8: We define filtrations $\check{\mathbb{I}}=\left(\check{\mathcal{I}}_{t}\right)_{t \in[0, T]}$ and $\widehat{\mathbb{I}}=\left(\widehat{\mathcal{I}}_{t}\right)_{t \in[0, T]}$, by letting $\check{\mathcal{I}}_{t}$ be trivial for $t \in[0, T), \check{\mathcal{I}}_{T}:=\mathcal{I}_{T}$, and $\widehat{\mathcal{I}}_{t}:=\mathcal{I}_{T}$ for all $t \in[0, T]$. Then the relations (4.17) hold. Let $\check{\mathbb{F}}$ and $\widehat{\mathbb{F}}$ be defined as in Proposition 4.7 and let the strategy space for the investor with information flow $\mathbb{H} \in\{\check{\mathbb{F}}, \mathbb{F}, \widehat{\mathbb{F}}\}$ be given by $\Theta(\mathbb{H}):=\Theta_{3}(\mathbb{H})$. The latter causes no loss of generality since $\Theta_{3}(\mathbb{F})$ 
satisfies (2.5) and leads to the same utility-indifference value $\pi(B, \mathbb{F})$ as any other $\Theta$ for which (2.5) holds. Proposition 4.7 then yields that the relations

$$
\pi(B ; \check{\mathbb{F}}) \geq \pi(B ; \mathbb{F}) \geq \pi(B ; \widehat{\mathbb{F}})
$$

hold between the utility-indifference prices for investors with information flows $\mathbb{H} \in\{\check{\mathbb{F}}, \mathbb{F}, \widehat{\mathbb{F}}\}$. Since $\check{\mathbb{F}}$ and $\widehat{\mathbb{F}}$ are piecewise constant, one can compute the foregoing bounds for $\pi(B ; \mathbb{F})$ via Theorem 4.4 (recalling the argument at the beginning of the proof for Prop. 4.7). This yields the claim.

Obviously, the presented valuation bounds would not help much if they were too far apart. The good message of the next example is that they in fact can be fairly close. Taking into account ever present issues like model uncertainly, the bounds might be sufficiently close to give some valuations in practice. If this is not the case, there is a straightforward way to compute improved bounds: Simply take lower and upper piecewise constant 'bounds' ( $\check{\mathbb{I}}$ and $\widehat{\mathbb{I}}$ ) for the information flow $\mathbb{I}$ under consideration, and then compute the valuation bounds from Proposition 4.7 via the computation scheme from Theorem 4.4. A natural choice would be $\check{\mathcal{I}}_{t}:=\mathcal{I}_{t_{k}}$ and $\widehat{\mathcal{I}}_{t}:=\mathcal{I}_{t_{k+1}}$ for $t \in\left[t_{k}, t_{k+1}\right)$; using a subdivision of $0=t_{0}<t_{1}<\cdots<t_{n}=T$ can further improve the valuation bounds.

Example 4.9 ('Financial Stop-Loss' reinsurance contract)

Let us consider an insurance company that seeks protection from a reinsurer against combined losses from its financial investments and the insured claims. To this end, the latter might offer a 'financial stop-loss contract' which covers a certain range of combined insurance losses $Y_{T}$ and losses from investments $\delta\left(\widetilde{S}_{0}-\widetilde{S}_{T}\right)$ in financial assets $\widetilde{S}$ (non-discounted), a stock index say, up to time $T$. That is, the contract pays at time $T$ in discounted terms the amount

$$
B:=e^{-r T} \min \left\{\left(Y_{T}+\delta\left(\widetilde{S}_{0}-\widetilde{S}_{T}\right)-K_{1}\right)^{+},\left(K_{2}-K_{1}\right)\right\}
$$

where $K_{1}<K_{2}<\infty$ are the layers of coverage, $r>0$ is the risk-less interest rate, and $\delta \geq 0$ quantifies the level of financial investment in the stock index $\widetilde{S}$.

The subsequent setup for this example is essentially borrowed from Møller (2003a), who however uses a valuation approach with respect to a different (quadratic) indifference criterion, derived from the standard deviation principle. As in the Black-Scholes model, the (discounted) stock index $S_{t}=e^{-r t} \widetilde{S}_{t}$ is supposed to evolve according to a geometric Brownian motion

$$
\frac{d S_{t}}{S_{t}}=(\gamma-r) d t+\sigma d W_{t}, \quad S_{0} \in(0, \infty)
$$

where $W$ is a standard Brownian motion, $\gamma-r \in \mathbb{R}$ is the excess return rate, and $\sigma>0$ is the volatility. Then

$$
S_{t}=S_{0} \exp \left(\sigma W_{t}+\left((\gamma-r)-\frac{1}{2} \sigma^{2}\right) t\right), \quad t \in[0, T]
$$

and $S_{T}$ is lognormally distributed. The insurance losses up to time $T$ are modelled by

$$
Y_{T}:=y_{0} \mathcal{E}(\kappa \widehat{W}+\mu t)_{T}=y_{0} \exp \left(\kappa \widehat{W}_{T}+\left(\mu-\frac{1}{2} \kappa^{2}\right) T\right)
$$


where $\mu \in \mathbb{R}, \kappa>0$, and $y_{0}>0$ are constants and $\widehat{W}$ is another Brownian motion. Of course, such a modelling clearly is a simplification. Suppose that $W$ and $\widehat{W}$ are correlated with coefficient $\varrho \in[-1,+1]$. For $|\varrho|<1$, the process $W_{t}^{\perp}:=\left(\sqrt{1-\varrho^{2}}\right)^{-1}\left(\widehat{W}_{t}-\varrho W_{t}\right)$ is well-defined, and $W$ and $W^{\perp}$ are independent $P$-Brownian motions. The setup fits in the framework of Sect. 4.1 with $\mathbb{F}^{0}:=\mathbb{F}^{W}, \mathbb{I}:=\mathbb{F}^{W^{\perp}}$ when $|\varrho|<1$, and with $\mathbb{I}$ being trivial when $|\varrho|=1$. The minimal entropy martingale measure $Q^{*}$ is given by

$$
\frac{d Q^{*}}{d P}=\mathcal{E}\left(-\frac{\gamma-r}{\sigma} W\right)_{T}=\exp \left(-\frac{\gamma-r}{\sigma} W_{T}-\frac{1}{2} \frac{(\gamma-r)^{2}}{\sigma^{2}} T\right)
$$

and $S$ satisfies $d S_{t} / S_{t}=\sigma d W_{t}^{*}$ under $Q^{*}$ where $W_{t}^{*}:=W_{t}+\frac{\gamma-r}{\sigma} t$ is a $Q^{*}$-Brownian motion. Note that $W^{\perp}$ and $W^{*}$ are independent $Q^{*}$-Brownian motions and $\varrho t=\langle W, \widehat{W}\rangle_{t}=\left\langle W^{*}, \widehat{W}\right\rangle_{t}$.

As concrete parameters, let $S_{0}=1, r=0.05, \sigma=0.25, \gamma=0.1, y_{0}=0.83, \kappa=0.15, \mu=0, \varrho=0$, $T=1, K_{1}=0.85$, and $K_{2}=1.15$, following Møller (2003a). In fact, this parameterization specifies the same probabilistic model as 'contract 1' in his Section 6.2. This allows for a first comparison of the two indifference valuation approaches. Figure 3 shows the upper and lower bounds (4.21) for

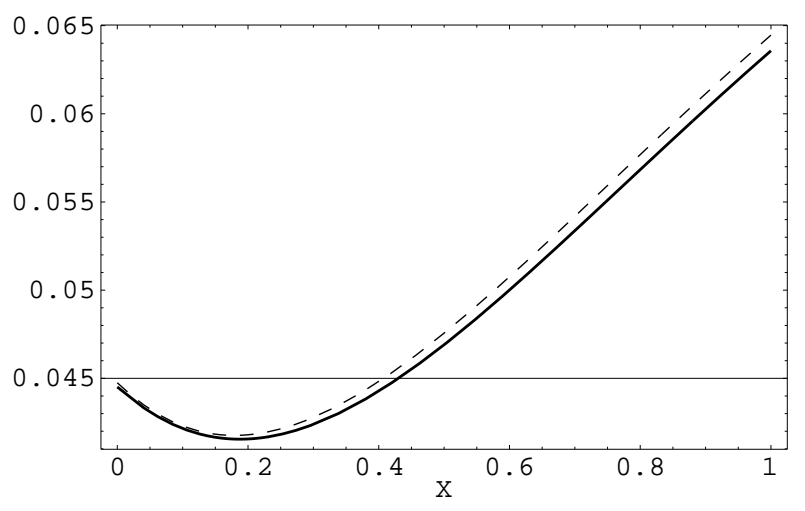

Figure 3: Upper (dashed line) and lower (solid line) bounds for the utility-indifference price of the financial stop loss contract $B$ as a function of $\delta$ ( $X$-axis)

the (exponential) utility-indifference price $\pi(B ; 1)$ of the claim $(4.24)$ as a function of the investment level $\delta$. Considering the contract value for zero investment at $\delta=0$, we see that the risk aversion $\alpha=1$ corresponds to a relative safety loading (cf. (3.10) and (4.5)) of

$$
\frac{\pi(B)}{E_{Q^{0}}[B]}-1=\frac{\pi(B)}{E^{*}[B]}-1 \approx \frac{0.045}{0.038}-1 \approx 18 \text { percent }
$$

This is comparable in size to the safety loading of the valuation principle used for Figure 3 in Møller (2003a), where the claim $B$ was valued by 0.048 for $\delta=0$. Our Figure 3 shows that the utility indifference price is minimized by an investment in the financial market with $\delta>0$. Intuitively, this reflects the fact that gains from the financial investments can offset the insurance losses, resulting in a sort of diversification bonus. The same effect shows up in Møller (2003a) but it is more pronounced here. At first glance, the utility-indifference price moreover achieves its minimum apparently for a higher investment level $\delta$, and leads to somewhat smaller premiums for $\delta>0$. Similar as in Møller 
(2003a), the valuation bounds are fairly close. In fact, the relative difference between our upper and lower bounds for the utility-indifference price is less than 2 percent for all $\delta \in[0,1]$ and about 0.5 percent for $\delta=0$. Overall, the two different indifference valuation approaches yield roughly comparable results here. A more detailed analysis however is clearly necessary and subject to future work. One should guess that the differences between the present utility criterion and a symmetric quadratic criterion as in Møller (2003a) would be much more pronounced when the distribution of the payoff is highly skewed (in its tails) - simply because a symmetric criterion punishes all deviations from the mean, gains as losses, in the same way.

\subsection{Additivity and diversification}

We already know that the mapping $B \mapsto \pi(B ; \alpha)$ is linear on the space of bounded attainable claims in general (see (3.8)). In the static situation which corresponds to setting $S \equiv 0$, the utility-indifference price $\pi(B ; \alpha)=1 / \alpha \log E_{P}[\exp (\alpha B)]$ is known to be additive with respect to independent claims, i.e., $\pi\left(B_{1}+B_{2}\right)=\pi\left(B_{1}\right)+\pi\left(B_{2}\right)$ holds for $P$-independent variables $B_{1,2}$ with sufficient integrability (see Gerber (1979), chapter 5 ). In general, $\pi$ is not additive (or sub-additive) even in the static situation.

In the present section, we are going to obtain similar results on additivity for the dynamic case. More precisely, we will show that the utility indifference price and the hedging strategy are additive when applied to a sum of claims which are conditionally $P$-independent given the information of the complete financial sub-market. Clearly, this can considerably facilitate the computations for an aggregated amount of claims since the problem is reduced to the level of individual claims. Using our additivity result, we then prove that diversification leads asymptotically to a risk-neutral valuation with respect to a large number of independent additional sources of risk.

Theorem 4.10 (Additivity) Suppose (2.5) holds. Let $B_{1}, B_{2} \ldots$ be such that each $B_{i}$ is $\mathcal{F}_{T}^{0} \vee \mathcal{I}_{T}^{i}-$ measurable and satisfies $\exp \left(\gamma\left|B_{i}\right|\right) \in L^{1}(P)$ for all $\gamma \in \mathbb{R}$. Then, for all $n \in \mathbb{N}$,

$$
\pi\left(\sum_{i=1}^{n} B_{i} ; \alpha\right)=\sum_{i=1}^{n} \pi\left(B_{i} ; \alpha\right) \quad \text { and } \quad \psi\left(\sum_{i=1}^{n} B_{i} ; \alpha\right)=\sum_{i=1}^{n} \psi\left(B_{i} ; \alpha\right) .
$$

Proof: It suffices to consider the case $n=2$ since the argument can be iterated. Let $Q^{i}:=Q^{B^{i}}$, $i=1,2$, and denote by $Z^{i}$ the $\mathbb{F}$-density process of $Q^{i}$ with respect to $Q^{*}$. We first show that $d \bar{Q}:=Z_{T}^{1} Z_{T}^{2} d Q^{*}$ defines an element of $\mathbb{P}_{e} . Z_{T}^{i}$ is $\mathcal{F}_{T}^{0} \vee \mathcal{I}_{T}^{i}$ measurable by Lemma 4.2. Hence $Z_{T}^{1}$ and $Z_{T}^{2}$ are $Q^{*}$-independent given $\mathcal{F}_{T}^{0}$, and we obtain

$$
E^{*}\left[Z_{T}^{1} Z_{T}^{2} \mid \mathcal{F}_{T}^{0}\right]=E^{*}\left[Z_{T}^{1} \mid \mathcal{F}_{T}^{0}\right] E^{*}\left[Z_{T}^{2} \mid \mathcal{F}_{T}^{0}\right]=1 \cdot 1=1
$$

using Lemma 4.3. So $\bar{Q}$ is a probability measure and $\bar{Q} \sim P$ since $Z_{T}^{i}>0$. By (4.4) and Lemma 4.3, 
we have $E^{*}\left[Z_{T}^{j} \mid \mathcal{F}_{T}^{0} \vee \mathcal{I}_{T}^{i} \vee \mathcal{I}_{t}^{j}\right]=E^{*}\left[Z_{T}^{j} \mid \mathcal{F}_{T}^{0} \vee \mathcal{I}_{t}^{j}\right]=Z_{t}^{j}$ for $i, j \in\{1,2\}$ with $i \neq j$, and

$$
\begin{aligned}
E^{*}\left[Z_{T}^{1} Z_{T}^{2} \mid \mathcal{F}_{t}\right] & =E^{*}\left[Z_{T}^{1} Z_{T}^{2} \mid \mathcal{F}_{t}^{0} \vee \mathcal{I}_{t}^{1} \vee \mathcal{I}_{t}^{2}\right] \\
& =E^{*}\left[Z_{T}^{1} E^{*}\left[Z_{T}^{2} \mid \mathcal{F}_{T}^{0} \vee \mathcal{I}_{T}^{1} \vee \mathcal{I}_{t}^{2}\right] \mid \mathcal{F}_{t}^{0} \vee \mathcal{I}_{t}^{1} \vee \mathcal{I}_{t}^{2}\right] \\
& =E^{*}\left[Z_{T}^{1} Z_{t}^{2} \mid \mathcal{F}_{t}^{0} \vee \mathcal{I}_{t}^{1} \vee \mathcal{I}_{t}^{2}\right] \\
& =Z_{t}^{2} E^{*}\left[E^{*}\left[Z_{T}^{1} \mid \mathcal{F}_{T}^{0} \vee \mathcal{I}_{t}^{1} \vee \mathcal{I}_{T}^{2}\right] \mid \mathcal{F}_{t}^{0} \vee \mathcal{I}_{t}^{1} \vee \mathcal{I}_{t}^{2}\right] \\
& =Z_{t}^{1} Z_{t}^{2} \quad \text { for } t \leq T
\end{aligned}
$$

Hence, $Z^{1} Z^{2}$ is the density process of $\bar{Q}$ with respect to $Q^{*}$. Using (4.4) and Lemma 4.3 gives

$$
E^{*}\left[Z_{t}^{1} Z_{t}^{2} \mid \mathcal{F}_{t}^{0} \vee \mathcal{I}_{s}\right]=E^{*}\left[Z_{t}^{1} \mid \mathcal{F}_{t}^{0} \vee \mathcal{I}_{s}\right] E^{*}\left[Z_{t}^{2} \mid \mathcal{F}_{t}^{0} \vee \mathcal{I}_{s}\right]=Z_{s}^{1} Z_{s}^{2}, \quad s \leq t \leq T,
$$

and we conclude via Lemma 4.3 that $\bar{Q}$ is in $\mathbb{P}_{e}$. We next show that $H(\bar{Q} \mid P)$ is finite. To this end, let $Z_{T}^{0}:=d Q^{*} / d P$ and consider

$$
\begin{aligned}
H(\bar{Q} \mid P) & =E^{*}\left[Z_{T}^{1} Z_{T}^{2} \log \left(Z_{T}^{0} Z_{T}^{1} Z_{T}^{2}\right)\right] \\
& =E^{*}\left[Z_{T}^{1} Z_{T}^{2} \log Z_{T}^{0}\right]+E^{*}\left[Z_{T}^{1} Z_{T}^{2} \log Z_{T}^{1}\right]+E^{*}\left[Z_{T}^{1} Z_{T}^{2} \log Z_{T}^{2}\right] .
\end{aligned}
$$

We prove that the three summands are all finite and thereby establish the last equality. By (4.28) the first summand equals $E^{*}\left[\log Z_{T}^{0}\right]=H\left(Q^{*} \mid P\right)<\infty$. Next $E^{*}\left[Z_{T}^{2} \mid \mathcal{F}_{T}^{0} \vee \mathcal{I}_{T}^{1}\right]=E^{*}\left[Z_{T}^{2} \mid \mathcal{F}_{T}^{0}\right]=1$ implies via conditioning on $\mathcal{F}_{T}^{0} \vee \mathcal{I}_{T}^{1}$ that the second addend equals $E^{*}\left[Z_{T}^{1} \log Z_{T}^{1}\right]=H\left(Q^{1} \mid Q^{*}\right)$. It follows from $H\left(Q^{1} \mid P\right) \geq H\left(Q^{1} \mid Q^{*}\right)+H\left(Q^{*} \mid P\right)$ (by Csiszár (1975), Theorem 2.2) and $Q^{1} \in \mathbb{P}_{f}$ that $H\left(Q^{1} \mid Q^{*}\right)<\infty$. Analogously, the third addend is equal to $H\left(Q^{2} \mid Q^{*}\right)$ and also finite.

Hence, $\bar{Q}$ is in $\mathbb{P}_{f} \cap \mathbb{P}_{e}$. Hölder's inequality yields $\exp \left(\gamma\left|B_{1}+B_{2}\right|\right) \in L^{1}(P)$ for any $\gamma \in \mathbb{R}$ since $\exp \left(2 \gamma\left|B_{i}\right|\right) \in L^{1}(P)$ by hypothesis. Recalling $Q^{*}=Q^{0}$, Part 1 of Corollary 3.6 gives

$$
\frac{d \bar{Q}}{d Q^{0}}=\frac{d Q^{1}}{d Q^{0}} \frac{d Q^{2}}{d Q^{0}}=\exp \left(-\alpha\left(\left(\pi\left(B_{1}\right)+\pi\left(B_{2}\right)\right)+\int_{0}^{T}\left(\psi\left(B_{1}\right)+\psi\left(B_{2}\right)\right) d S-\left(B_{1}+B_{2}\right)\right)\right)
$$

and by part 2 of Corollary 3.6 this yields the claim.

As an application of the previous theorem, we are going to show that diversification leads asymptotically to a risk-neutral valuation with respect to a large number of independent sources $\mathbb{I}^{k}$ of risk. This is intuitively very plausible: The further (non-tradable) sources of uncertainty in the claim are not hedged by dynamical trading but in a way by the law of large numbers. To explain the contribution of the next result let us first introduce some notation. Let $\left(N_{t}^{i}\right)_{t \in[0, T]}, i=1,2, \ldots$, be processes (with RCLL-paths) which are i.i.d. and independent of $\mathcal{F}_{T}^{0}$ under $P$, and let

$$
\mathbb{I}^{i}:=\mathbb{F}^{N^{i}} .
$$

Each $\left(S, N^{i}\right)$ has right continuous paths with left limits. Let $f$ be a fixed real-valued measurable function on this path-space (with respect to the Skohorod topology) and let the claims be given as

$$
B_{i}:=f\left(S, N^{i}\right) \quad, i \in \mathbb{N} .
$$


This means that claims can depend on the whole evolution of $S$ and $N$. To explain the crucial difference between the intuitive law of large numbers argument from above and the subsequent corollary, suppose for the moment that $f$ is bounded. The intuitive reasoning above then argues that $\frac{1}{n} \sum_{i=1}^{n} B_{i}$ converges a.s. to $\bar{B}:=E^{*}\left[f\left(S, N^{1}\right) \mid \mathcal{F}_{T}^{0}\right]$ by a conditional version of the law of large numbers. This limit can be interpreted as follows: By diversifying his portfolio to more but smaller claims, the issuer can eliminate the risk associated with the uncertainty about the outcomes of the $N^{i}$ 's. Note that such diversification still leaves all risk which stems from the uncertainty about the future evolution of the asset prices $S$ at the issuer, since all claims $B_{i}$ are linked to the same $S$. However, as the tradable assets $S$ span a complete market, the remaining risk can be perfectly hedged and its price is determined by No-Arbitrage arguments as the replication cost! Technically, this completeness corresponds to the $\left(Q^{*}, \mathbb{F}^{0}\right)$-representation property of $S$, and by (3.8) we must have $\pi(\bar{B} ; \alpha)=E^{*}[\bar{B}]$. Summing up, we have

$$
\pi\left(\lim _{n \rightarrow \infty} \frac{1}{n} \sum_{i=1}^{n} B_{i} ; \alpha\right)=E^{*}[\bar{B}],
$$

i.e. $E^{*}[\bar{B}]$ is the indifference price of the aggregated portfolio of claims $\bar{B}$ that represents the case of "infinite diversification". In comparison to this, the subsequent corollary proves that the utility-indifference price of the "finitely diversified" portfolio $\frac{1}{n} \sum_{i=1}^{n} B_{i}$ itself tends to $E^{*}[\bar{B}]$ if the diversification increases; i.e.

$$
\lim _{n \rightarrow \infty} \pi\left(\frac{1}{n} \sum_{i=1}^{n} B_{i} ; \alpha\right)=E^{*}[\bar{B}] .
$$

In reality, an investor can diversify his risks only by holding a large but finite portfolio. Therefore the latter limit is the one that is relevant for answering the question: How does increasing diversification asymptotically affect the indifference price of the overall portfolio of claims? It turns out that both limits coincide in the present setting. In this sense, the following result justifies the conclusion of the intuitive reasoning outlined above.

Theorem 4.11 (Diversification) Suppose (4.29) and (2.5) hold. Let $B_{1}, B_{2}, \ldots$ be given by (4.30) and suppose that $\exp \left(\gamma\left|B_{1}\right|\right) \in L^{1}(P)$ for all $\gamma \in \mathbb{R}$, and $B_{1} \in L_{\exp }\left(Q^{*}\right)$. Then

$$
\pi\left(\frac{1}{n} \sum_{i=1}^{n} B_{i} ; \alpha\right) \stackrel{n \rightarrow \infty}{\longrightarrow} E^{*}\left[B_{1}\right] .
$$

By (4.4), the limit in (4.31) takes the form $E^{*}[h(S)] E_{P}\left[g\left(N^{1}\right)\right]$ when $B_{1}=f\left(S, N^{1}\right)=h(S) g\left(N^{1}\right)$ for measurable functions $g$ and $h$.

Proof: By symmetry, we have $\pi\left(f\left(S, N^{i}\right) / n ; \alpha\right)=\pi\left(f\left(S, N^{1}\right) / n ; \alpha\right)$ for all $i$. By Theorem 4.10, (3.4) and Proposition 3.2 we then obtain

$$
\pi\left(\frac{1}{n} \sum_{i=1}^{n} B_{i} ; \alpha\right)=n \pi\left(\frac{f\left(S, N^{1}\right)}{n} ; \alpha\right)=\pi\left(f\left(S, N^{1}\right) ; \frac{\alpha}{n}\right) \stackrel{n \rightarrow \infty}{\longrightarrow} E_{Q^{0}}\left[f\left(S, N^{1}\right)\right] .
$$

Since $Q^{0}=Q^{*}$, this yields the claim. 
We illustrate the result by giving a simple example in the context of equity-linked life insurance. In the literature, it is common to model the equity prices by a (generalized) Black-Scholes model and to consider the mortality risk as being $P$-independent from $S$, and it is typically argued that equity linked payments which are conditional on the survival or death of a policy holder should be valued by the Black-Scholes value of the unconditional payoffs multiplied with the $P$-probabilities of survival or death, respectively, see e.g. Brennan and Schwarz (1979) or Rolski et al. (1998) and references therein. The intuitive reasoning for this is basically that the mortality risks in the individual policies are eliminated by a law of large numbers. Hence, they are practically not present for the insurance company. To make this heuristic motivation more rigorous, let us apply the utility based pricing for an aggregated portfolio of insurance policies.

\section{Example 4.12 (Equity-linked life insurances)}

We consider the (discounted) standard Black-Scholes model $d S_{t}=S_{t}\left(\gamma d t+\sigma d W_{t}\right)$ with constant coefficients $\gamma \in \mathbb{R}$ and $\sigma>0$. Let $N^{1}, N^{2}, \ldots$ be i.i.d. Poisson processes with deterministic intensities $\lambda(t)$, independent of $W$, and suppose that the information flow $\mathbb{F}$ is generated by $W, N^{1}, N^{2}, \ldots$. The process $N^{i}$ models the survival of policy holder $i$ who is thought to be alive until the first jump of $N^{i}$ occurs. We consider claims of the form $B_{i}=h(S) I_{\left\{N_{T}^{i}=0\right\}}$ with $h$ measurable and bounded, e.g. $h(S)=K_{1}+\left(S_{T}-K_{1}\right)^{+} \wedge\left(K_{2}-K_{1}\right)$ with constants $K_{1}<K_{2}$. Theorem 4.11 yields

$$
\lim _{n \rightarrow \infty} \pi\left(\frac{1}{n} \sum_{i=1}^{n} h(S) I_{\left\{N_{T}^{i}=0\right\}}\right)=E^{*}\left[h(S) I_{\left\{N_{T}^{1}=0\right\}}\right]=E^{*}[h(S)] P\left[N_{T}^{1}=0\right] .
$$

This means that for a large number of policy holders with small individual contracts the utilityindifference price of the aggregated portfolio of insurance claims tends to a valuation which is risk-neutral with respect to mortality risk, although the issuer has absolute risk aversion $\alpha>0$ and mortality risk has been assumed to be non-tradable. So, the utility based argument approves and is in accordance with a commonly used practice (see, e.g., Rolski et al. (1998)). Just for comparison, let us note that mortality risk would have to be modelled as a part of the financial market $S$ if it should be considered as a (liquidly) tradable asset, e.g. as in Sondermann (1991).

\section{A Appendix}

This section contains more technical results and proofs which are omitted from the main body of the paper. Some are rather straightforward modifications of existing proofs, and are just included for the reader's convenience.

Lemma A.1 Assume (2.4). Then (2.3) holds, $B$ is in $L^{1}(Q)$ for all $Q \in \mathbb{P}_{f}$, and

$$
H(Q \mid P)=E_{Q}\left[\log \frac{d Q}{d P_{B}}+\log \frac{e^{\alpha B}}{E\left[e^{\alpha B}\right]}\right]=H\left(Q \mid P_{B}\right)+\log \frac{1}{E\left[e^{\alpha B}\right]}+E_{Q}[\alpha B] .
$$

Proof: First, (2.4) implies $\exp (\varepsilon|B|) \in L^{1}(P)$. By Lemma 8 in Delbaen et al. (2002) we have

$$
E_{Q}[\varepsilon|B|] \leq H(Q \mid P)+\frac{1}{e} E_{P}\left[e^{\varepsilon|B|}\right] \quad \text { for } Q \ll P \text {. }
$$


Using (2.4), we obtain that $B \in L^{1}(Q)$ for $Q \in \mathbb{P}_{f}(P)$. This establishes the second equality in (A.1) for $Q \in \mathbb{P}_{f}(P)$ while the first equality holds by the definition of $P_{B}$. We conclude that $\mathbb{P}_{f}(P) \subseteq \mathbb{P}_{f}\left(P_{B}\right)$. For the converse inclusion, Lemma 8 in Delbaen et al. (2002) yields that (A.2) also holds with $P_{B}$ instead of $P$. Thus, we obtain that $E_{Q}[\varepsilon|B|]<\infty$ for $Q \in \mathbb{P}_{f}\left(P_{B}\right)$ since

$$
E_{P_{B}}\left[e^{\varepsilon|B|}\right]=\text { const } E_{P}\left[e^{\alpha B+\varepsilon|B|}\right] \leq \operatorname{const} E_{P}\left[e^{(\alpha+\varepsilon) B^{+}+\varepsilon B^{-}}\right]
$$

and the right hand side is finite by hypothesis (2.4). We conclude that (A.1) also holds for $Q \in$ $\mathbb{P}_{f}\left(P_{B}\right)$, and this implies the converse inclusion $\mathbb{P}_{f}\left(P_{B}\right) \subseteq \mathbb{P}_{f}(P)$.

Proof of Proposition 2.2: The exponential utility maximization problem (2.7) with terminal liability $B$ reduces to the ordinary utility maximization problem

$$
E\left[e^{\alpha B}\right] \sup _{\vartheta \in \Theta} E_{P_{B}}\left[-\exp \left(-\alpha\left(x+\int_{0}^{T} \vartheta d S\right)\right)\right]
$$

without terminal liability but with $P_{B}$ instead of $P$ by a measure transformation, namely

$$
-\exp \left(-\alpha\left(x+\int_{0}^{T} \vartheta d S-B\right)\right)=-\exp \left(-\alpha\left(x+\int_{0}^{T} \vartheta d S\right)\right)\left(E\left[e^{\alpha B}\right] \frac{d P_{B}}{d P}\right) .
$$

Furthermore, (A.1) shows that

$$
\sup _{Q \in \mathbb{P}_{f}}\left\{E_{Q}[\alpha B]-H(Q \mid P)\right\}+\log \frac{1}{E\left[e^{\alpha B}\right]}=\sup _{Q \in \mathbb{P}_{f}}\left\{-H\left(Q \mid P_{B}\right)\right\},
$$

so that both suprema are attained by the same $Q^{B}$ if the suprema are maxima. Recall that un$\operatorname{der}(2.1)$ and $\mathbb{P}_{f}\left(P_{B}\right) \cap \mathbb{P}_{e} \neq \emptyset$ the optimum $Q^{B}$ exists, and its density has the form $d Q^{B}=$ $\exp \left(-\alpha\left(\right.\right.$ const $\left.\left.+\left(\vartheta^{B} \cdot S\right)_{T}\right)\right) d P_{B}$ with $\vartheta^{B} \in L(S)$ such that $\vartheta^{B} \cdot S$ is a $Q^{B}$-martingale (see Corollary 2.1 of Frittelli (2000b) and Proposition 3.2 and the proof of Theorem 4.3 of Grandits and Rheinländer (2002)). This gives part 1 and the first half of part 2. Now we can apply Theorem 2 of Delbaen et al. (2002) (in the extended form due to Kabanov and Stricker (2002)) to the optimization problem (A.3) with $\Theta$ given by $\tilde{\Theta}_{2}:=\Theta_{\mathcal{M}} \cap\left\{\vartheta \in L(S) \mid e^{-\alpha(\vartheta \cdot S)_{T}} \in L^{1}\left(P_{B}\right)\right\}$. Rewriting the results with respect to $P$ instead of $P_{B}$ then yields all claims of Proposition 2.2 with $\Theta=\tilde{\Theta}_{2}$. Note that $\Theta_{\mathcal{M}}$ contains $\tilde{\Theta}_{2}$ and in addition only suboptimal strategies which yield expected utility $-\infty$. Thus, the results also hold for $\Theta=\Theta_{\mathcal{M}}$ and therefore for any $\Theta$ which satisfies (2.5). Note that the second equality in part 4 follows by part 2 .

Next, we show that Proposition 3.5 restates a result of Grandits and Rheinländer (2002).

Proof of Proposition 3.5: Assume $P_{B}$ is well defined. Grandits and Rheinländer (2002) showed that a measure $\bar{Q} \in \mathbb{P}_{a}$ minimizes $H\left(Q \mid P_{B}\right)$ over $Q \in \mathbb{P}_{a}$ if its density has the form

$$
\frac{d \bar{Q}}{d P_{B}}=\exp \left(-\alpha\left(c+\int_{0}^{T} \bar{\vartheta} d S\right)\right) \quad \text { for some } c \in \mathbb{R} \text { and } \bar{\vartheta} \in L(S)
$$


and one of the following two conditions holds:

(i) $\bar{\vartheta} \cdot S$ is a $\bar{Q}-\mathcal{B M O}$-martingale and $e^{\varepsilon \alpha \int_{0}^{T} \bar{\vartheta} d S} \in L^{1}\left(P_{B}\right)$ for some $\varepsilon>0$, or

(ii) $\bar{Q} \in \mathbb{P}_{f}$ and $\bar{\vartheta} \in \Theta_{\mathcal{M}}$.

Note that $(A .6)$ in combination with (i) or (ii) already implies that $\bar{Q} \in \mathbb{P}_{f} \cap \mathbb{P}_{e}$ since $d \bar{Q} / d P_{B}>0$ and $H\left(\bar{Q} \mid P_{B}\right)=-\alpha c<\infty$. Variant (i) is just a re-draft of Proposition 3.4 and variant (ii) readily follows from Proposition 3.2, both in Grandits and Rheinländer (2002). Rewriting these results with respect to $P$ instead of $P_{B}$ then yields the formulation of our Proposition 3.5.

For our modelling in Section 4 we needed

Lemma A.2 The filtrations $\mathbb{F}$ and $\mathbb{I}$ in the setting of Section 4.1 satisfy the usual conditions.

Proof: It suffices to show right-continuity. We extend an idea from He and Wang (1982) to the case when there are countably many factors of additional risk, and show the argument just for $\mathbb{I}$ : Let $A_{i} \in \mathcal{I}_{T}^{i}, i=1, \ldots, n$, for some $n \in \mathbb{N}$. By (4.4), we have $E\left[\prod_{i=1}^{n} 1_{A_{i}} \mid \mathcal{I}_{s}\right]=\prod_{i=1}^{n} E\left[1_{A_{i}} \mid \mathcal{I}_{s}^{i}\right]$ for any $s \in[0, T]$. Letting $s \downarrow t$ for some $t \in[0, T]$ and using (4.4) and the usual conditions for the $\mathbb{I}^{i}$ 's then yields

$$
E\left[\prod_{i=1}^{n} 1_{A_{i}} \mid \mathcal{I}_{t+}\right]=\prod_{i=1}^{n} E\left[1_{A_{i}} \mid \mathcal{I}_{t+}^{i}\right]=\prod_{i=1}^{n} E\left[1_{A_{i}} \mid \mathcal{I}_{t}^{i}\right]=E\left[\prod_{i=1}^{n} 1_{A_{i}} \mid \mathcal{I}_{t}\right] .
$$

By monotone class arguments, this implies $E\left[f \mid \mathcal{I}_{t+}\right]=E\left[f \mid \mathcal{I}_{t}\right]$ for all bounded $\mathcal{I}_{T}$-measurable functions $f$ and therefore $\mathcal{I}_{t+}=\mathcal{I}_{t}$.

\section{References}

Amendinger, J., Becherer, D., Schweizer, M., 2003. A monetary value of initial information in portfolio optimization. Finance and Stochastics 7, 29-46.

Artzner, P., Delbaen, F., Eber, J.-M., Heath, D., 1999. Coherent measures of risk. Mathematical Finance 9 (3), 203-228.

Baxter, M., Rennie, A., 1996. Financial Calculus. Cambridge University Press, Cambridge.

Becherer, D., 2001a. The numeraire portfolio for unbounded semimartingales. Finance and Stochastics $5,327-341$.

Becherer, D., 2001b. Rational hedging and valuation with utility-based preferences. Ph.D. thesis, Technical University of Berlin, E-published: edocs.tu-berlin.de/diss/2001/becherer_dirk.htm.

Becherer, D., 2002. Utility-indifference hedging and valuation via reaction diffusion systems. To appear in Proceedings of The Royal Society, Series A . 
Bernoulli, D., 1738. Specimen theoriae novae de mensara sortis (exposition of a new theory on the measurement of risk). Comentarii Academiae Scientiarum Imperialis Petropolitanae as translated and reprinted in 1954, Econometrica (22), 23-36 .

Brennan, M., Schwarz, E., 1979. Pricing and Investment Strategies for Guaranteed Equity-linked Life Insurance. Huebner Foundation for Insurance Education, Wharton School, University of Pennsylvania, Philadelphia.

Bühlmann, H., 1983. The general economic premium principle. ASTIN Bulletin 14, 13-21.

Bühlmann, H., 1987. Actuaries of the third kind? ASTIN Bulletin 17, 137-138.

Csiszár, I., 1975. I-Divergence geometry of probability distributions. Annals of Probability 3, 146158.

Cvitanić, J., Schachermayer, W., Wang, H., 2001. Utility maximization in incomplete markets with random endowment. Finance and Stochastics 5, 259-272.

Davis, M., 1997. Option pricing in incomplete markets. In: Dempster, M. A. H., Pliska, S. R. (Eds.), Mathematics of Derivative Securities. Cambridge University Press, Cambridge, pp. 227-254.

Davis, M., 2000. Optimal hedging with basis risk. Preprint, Vienna University of Technology.

Delbaen, F., Grandits, P., Rheinländer, T., Samperi, D., Schweizer, M., Stricker, C., 2002. Exponential hedging and entropic penalties. Mathematical Finance 12, 99-123.

Delbaen, F., Hazendonck, J., 1989. A martingale approach to premium calculation principles in an arbitrage free market. Insurance: Mathematics and Economics 8, 269-277.

Delbaen, F., Schachermayer, W., 1994. A general version of the fundamental theorem of asset pricing. Mathematische Annalen 300, 463-520.

Duffie, D., Skiadas, C., 1994. Continuous-time security pricing, a utility gradient approach. Journal of Mathematical Economics 23, 107-131.

Embrechts, P., 2000. Actuarial versus financial pricing in insurance. Journal of Risk Finance 1, $17-26$.

Föllmer, H., Schied, A., 2002. Convex measures of risk and trading constraints. Finance and Stochastics 6, 429-447.

Frittelli, M., 2000a. Introduction to a theory of value coherent with the no-arbitrage principle. Finance and Stochastics 4 (1), 275-297.

Frittelli, M., 2000b. The minimal entropy martingale measure and the valuation problem in incomplete markets. Mathematical Finance 10 (1), 39-52. 
Geman, H., 1999. Learning about risk: Some lessons from insurance. European Finance Review 2, $113-124$.

Gerber, H., 1979. An Introduction to Mathematical Risk Theory. Huebner Foundation for Insurance Education, Wharton School, University of Pennsylvania, Phiadelphia.

Gerber, H., Parfumi, G., 1998. Utility functions: From risk theory to finance. North American Actuarial Journal 2, 74-91.

Gerber, H., Shiu, E., 1996. Actuarial bridges to dynamic hedging and option pricing. Insurance: Mathematics and Economics 18, 183-218.

Grandits, P., Rheinländer, T., 2002. On the minimal entropy martingale measure. Annals of Probability $30,1003-1038$.

He, S., Wang, J., 1982. The property of predictable representation of the sum of two semimartingales. Zeitschrift für Wahrscheinlichkeitstheorie und verwandte Gebiete 61, 141-152.

Henderson, V., 2002. Valuation of claims on non-traded assets using utility maximization. Mathematical Finance 12, 351-373.

Henderson, V., Hobson, D., 2002. Real options with constant relative risk aversion. Journal of Economic Dynamics and Control 27, 329-355.

Hodges, S. D., Neuberger, A., 1989. Optimal replication of contingent claims under transaction costs. Review of Futures Markets 8, 222-239.

Ihara, S., 1993. Information Theory for Continuous Systems. World Scientific, Singapore.

Jacod, J., 1979. Calcul Stochastique et Problèmes de Martingales. Lecture Notes in Mathematics 714. Springer, Berlin.

Kabanov, Y., Stricker, C., 2001. On equivalent martingale measures with bounded densities. In: Azéma, J., et al. (Eds.), Séminaire de Probabilits XXXV, Lecture Notes in Mathematics 1755. Springer, Berlin, pp. 139-148.

Kabanov, Y., Stricker, C., 2002. The optimal portfolio for the exponential utility maximization: Remarks to the six-authors paper. Mathematical Finance 12, 125-134.

Klüppelberg, C., 2001. Developments in insurance mathematics. In: Engquist, B., Schmid, W. (Eds.), Mathematics Unlimited - 2001 and Beyond. Springer, Berlin, pp. 703-722.

Kramkov, D., 1996. Optional decomposition of supermartingales and hedging contingent claims in incomplete security markets. Probability Theory and Related Fields 105, 459-479.

Mémin, J., 1980. Espaces de semi-martingales et changement de propabilité. Zeitschrift für Wahrscheinlichkeitstheorie und verwandte Gebiete 52, 9-39. 
Møller, T., 2001. On transformations of actuarial valuation principles. Insurance: Mathematics \& Economics 28, 281-303.

Møller, T., 2003a. Indifference pricing of insurance contracts: Applications. Insurance: Mathematics and Economics, to appear .

Møller, T., 2003b. Indifference pricing of insurance contracts in a product space model. Finance and Stochastics 7, 197-217.

Musiela, M., Zariphopoulou, T., 2002a. Indifference prices and related measures. Preprint, BNP Paribas / University of Austin.

Musiela, M., Zariphopoulou, T., 2002b. Optimal investment and pricing in incomplete markets: One-period binomial model case. Preprint, BNP Paribas / University of Austin.

Owen, M. P., 2002. Utility based optimal hedging in incomplete markets. Annals of Applied Probability $12,691-709$.

Rogers, L. C. G., 2001. Duality in constrained optimal investment and consumption problems: A synthesis. CIRANO lecture notes, available from http://www.statslab.cam.ac.uk/ ${ }^{\sim}$ chris/.

Rolski, T., Schmidli, H., Schmidt, V., Teugels, J., 1998. Stochastic Processes for Insurance and Finance. Wiley, New York.

Rouge, R., El Karoui, N., 2000. Pricing via utility maximization and entropy. Mathematical Finance $10,259-276$.

Schachermayer, W., 2001. Optimal investments when wealth may become negative. Annals of Applied Probability 11, 694-734.

Schachermayer, W., 2002. How potential investments may change the optimal portfolio for the exponential utility. Preprint, Vienna University of Technology.

Schweizer, M., 2001. From actuarial to financial valuation principles. Insurance: Mathematics \& Economics 28, 31-47.

Sondermann, D., 1991. Reinsurance in arbitrage-free markets. Insurance: Mathematics and Economics 10, 191-202.

Stricker, C., 2002. On the utility indifference price. Preprint, Université de Franche-Comté, to appear in the Proceedings of the 4th Ascona Conference 2002.

Young, V., Zariphopoulou, T., 2002. Pricing dynamic insurance risks using the principle of equivalent utility. Scandinavian Actuarial Journal , $246-279$. 


\section{Figures}

(As requested in the journal's instructions for authors, we provide each of the paper's figures additionally on a single page in this appendix.)

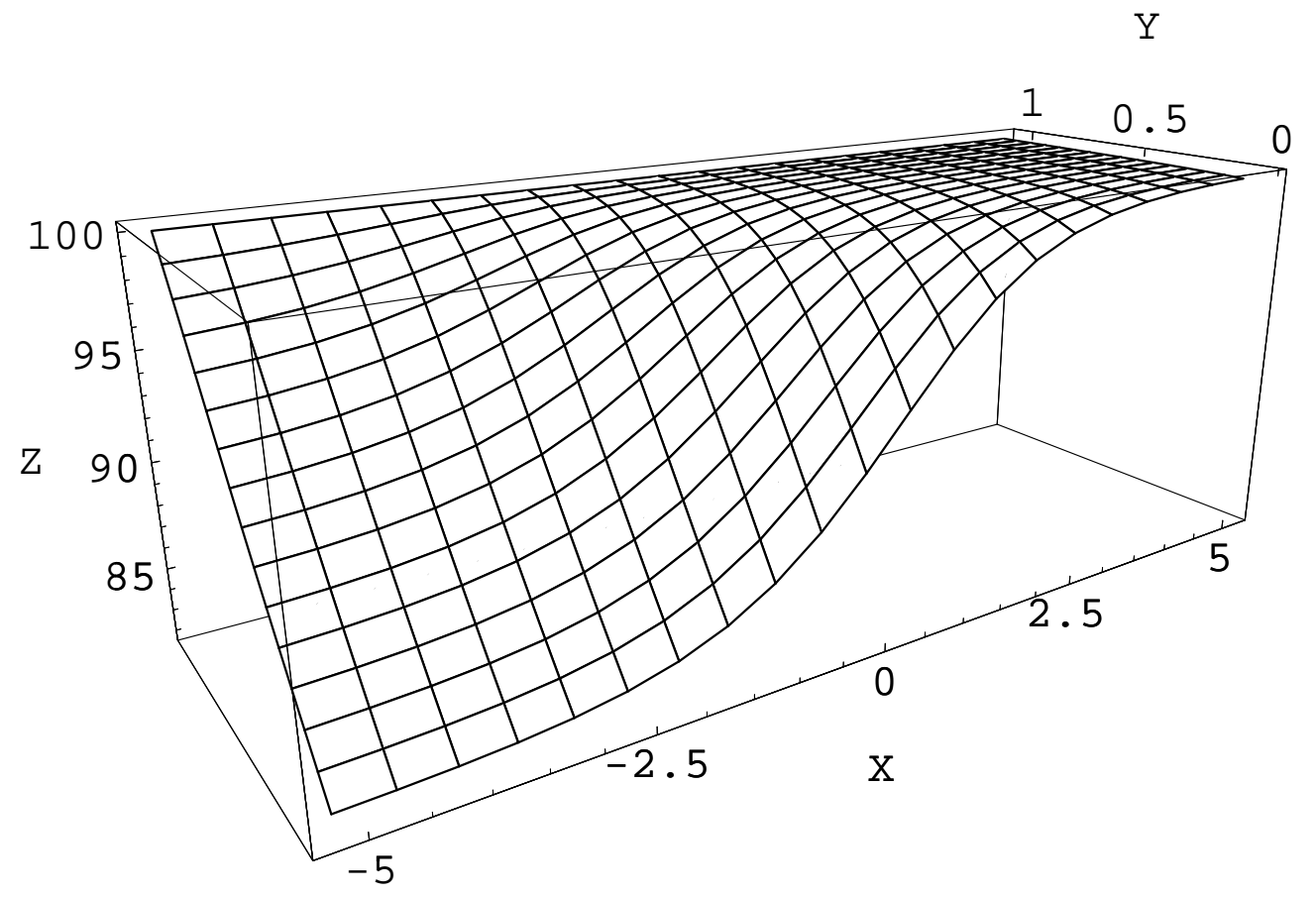




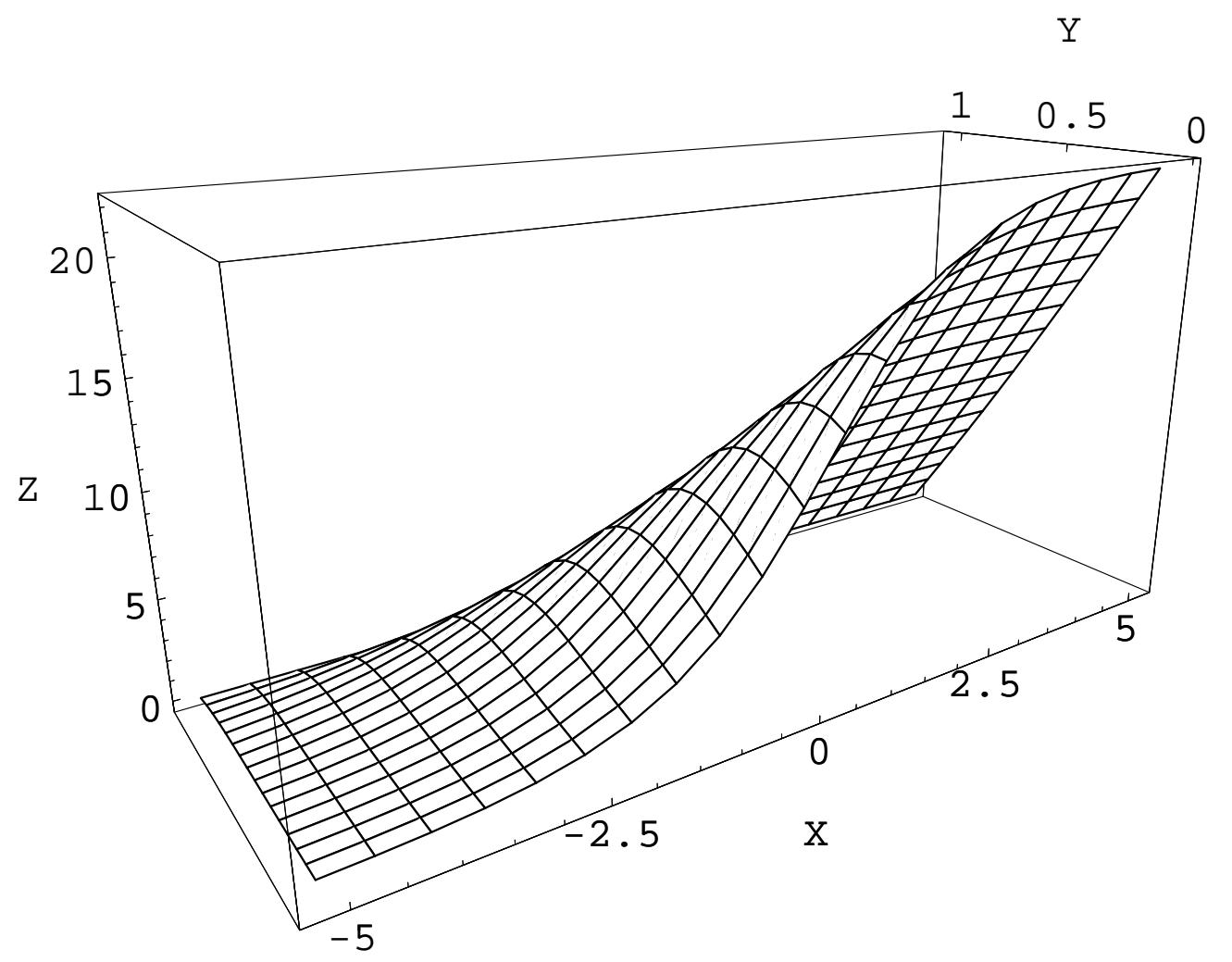




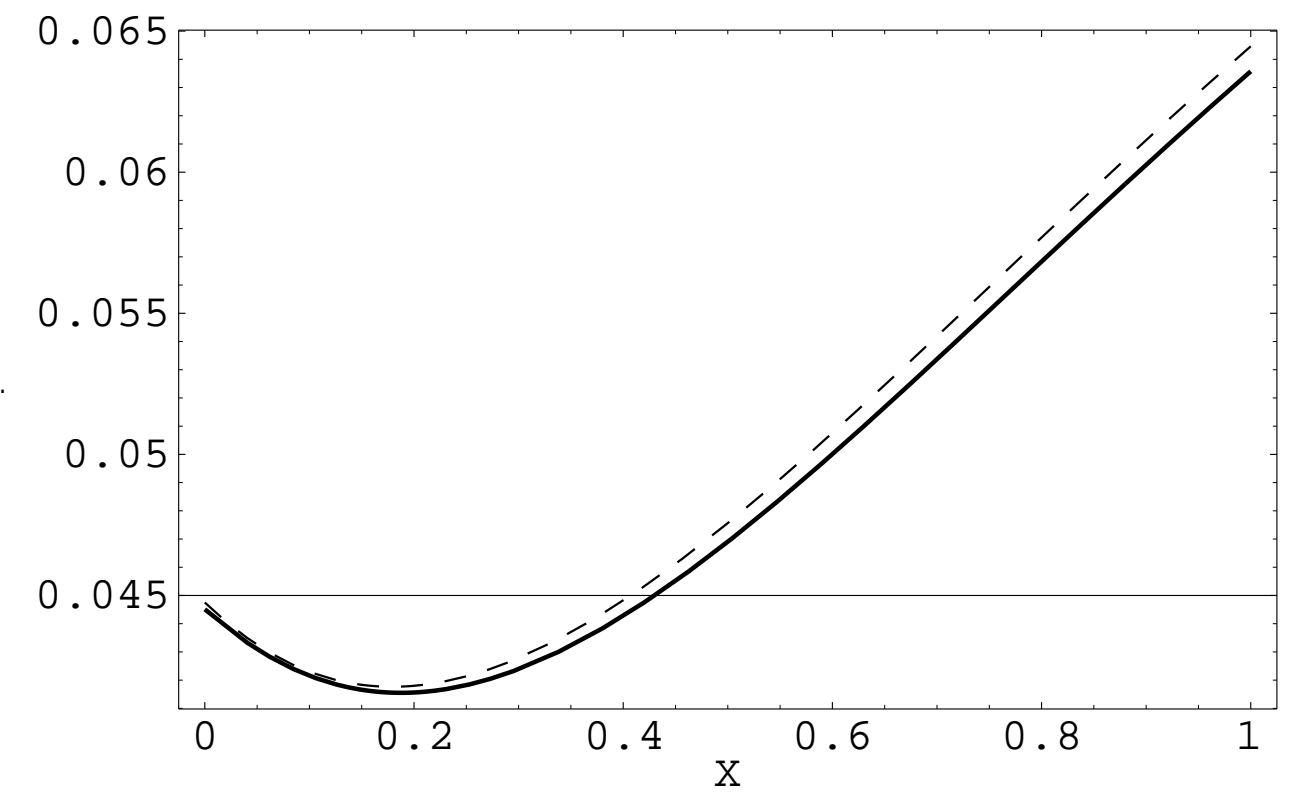

